\title{
Discrete Weierstrass transform in discrete Hermitian Clifford analysis
}

\author{
A. Masséa ${ }^{a, *}$, F. Sommen ${ }^{a}$, H. De Ridder ${ }^{a}$, T. Raeymaekers ${ }^{a}$ \\ ${ }^{a}$ Clifford Research Group, Department of Electronics and Information Systems, Ghent \\ University, Krijgslaan 281, 9000 Ghent, Belgium
}

\begin{abstract}
The classical Weierstrass transform is an isometric operator mapping elements of the weighted $L_{2}-$ space $\mathcal{L}_{2}\left(\mathbb{R}, \exp \left(-x^{2} / 2\right)\right)$ to the Fock space. It has numereous applications in physics and applied mathematics. In this paper, we define an analogue version of this transform in discrete Hermitian Clifford analysis, where functions are defined on a grid rather than the continuous space. This new transform is based on the classical definition, in combination with a discrete version of the Gaussian function and discrete counterparts of the classical Hermite polynomials. Furthermore, a discrete Weierstrass space with appropriate inner product is constructed, for which the discrete Hermite polynomials form a basis. In this setting, we also investigate the behaviour of the discrete delta functions and check if they are elements of this newly defined Weierstrass space.
\end{abstract}

Keywords: Discrete Clifford analysis, Weierstrass transform, Hermite polynomials, Weierstrass space, Delta functions

\section{Introduction}

The Weierstrass transform (or Gauss transform, Gauss-Weierstrass transform, [1]), named after Karl Weierstrass is one of the many convolution transforms in functional analysis. It averages the values of a function $f: \mathbb{R} \rightarrow \mathbb{R}$, by making the convolution with a Gaussian function to obtain a 'smoothed' version of $f$. Evaluating the smoothed function at the point $x$, points in the original function close to $x$ are given a higher weight, while points that are further away from $x$ get lower weights. It can be applied to solve the heat equation in one dimension: the Weierstrass transform $\mathcal{W}[f]$ gives the temperature distribution

\footnotetext{
* Corresponding author

Email addresses: Astrid.Masse@ugent.be (A. Massé), Franciscus.Sommen@ugent.be (F. Sommen), Hilde.DeRidder@ugent.be (H. De Ridder), Tim.Raeymaekers@ugent.be (T. Raeymaekers)
} 
of an infinitely long rod, one time unit later than the initial temperature given by the function $f$. In image processing, the transform is also known as the Gaussian blur, which helps to reduce the level of noise in the image. In signal processing, it is a simple kind of low-pass filter, whereas in statistics, it is used as a kernel density estimation. The Weierstrass transform is clearly related to various Fourier type transforms and it is a major part of (discrete) transform analysis in general. Applications are thus widely around.

This article is to be situated in the context of discrete Hermitian Clifford analysis, where a discrete version of the Weierstrass transform is constructed. Clifford analysis is a generalisation of classical analysis, in which null solutions of the Dirac operator play the key role. On the one hand, it can be viewed as a higher-dimensional theory of holomorphic functions in the complex plane. On the other hand, as the Dirac operator factorises the Laplacian, it is also a refinement of classical harmonic analysis. For further information about continuous Clifford analysis, we refer to [2]. Discrete Clifford analysis is a recent branch in functional analysis, which originated as an appropriate framework for the development of discrete counterparts of the function theory in classical Clifford analysis. Both numerical problems and theoretical results have been studied (see for example $[3,4,5,6,7,8]$ ). We will continue working in the discrete Clifford algebra model described in for example [9, 10, 11], where both forward and backward differences are introduced and combined with forward and backward basis elements. It should be noted that other discrete counterparts for the Weierstrass transform have been studied, see e.g. [12] and [13], which is used to approximate discrete data sets. Whereas these transforms are scalar valued, we use Clifford valued functions and the Clifford framework, where we can use the tools in this theory such as the factorisation of the Laplace operator. While, in this paper, we will restrict ourselves to the one dimensional case, discrete Clifford analysis is a theory that is well understood in higher dimensions. This will allow us to develop a higher dimensional Weierstrass transform in future work.

Together with a discrete Weierstrass transform, a discrete Weierstrass space will be constructed. The main idea is to use the discrete Gauss distribution $G$ as a weight function and the discrete Hermite polynomials as basis elements of the space. We ask ourselves if the delta-functions, the building blocks of discrete function theory, are elements of this space. For the discrete Weierstrass transform of a discrete function $f \in \mathcal{W}$, we use the discrete Gauss distribution $G$ together with the convolution kernel $\exp \left(-z^{2} / 2+\xi z\right)$, resulting in a direct analogue to the continuous Weierstrass transform.

This paper is organised as follows. In Section 2, we set up the preliminaries and describe the discrete Clifford setting in more detail. In Section 3, we define the discrete Weierstrass transform and continue to the definition of the discrete Weierstrass space in Section 4. In Section 5, we investigate the building blocks of discrete function theory, the $\delta$-functions, and ask whether they are elements of the discrete Weierstrass space. In this paper, we limit ourselves to the one- 
dimensional case.

\section{Preliminaries}

\subsection{Clifford analysis}

Clifford analysis - basis elements. Consider the $m$-dimensional real vector space $\mathbb{R}^{m}$ spanned by the orthonormal basis $\left\{e_{1}, \ldots, e_{m}\right\}$, endowed with a non-degenerate quadratic form with signature $(p, q), p+q=m$. The algebra generated by $e_{j}, j=1, \ldots, m$, satisfies the relations

$$
\begin{aligned}
e_{j}^{2}=1, & j=1, \ldots p, \\
e_{j}^{2}=-1, & j=p+1, \ldots, m, \\
e_{j} e_{k}+e_{k} e_{j}=0, & j \neq k, j, k=1, \ldots, m .
\end{aligned}
$$

The Clifford algebra thus constructed is denoted by $\mathbb{R}_{p, q}$. Denote for the set $A=\left\{j_{1}, \ldots, j_{r}\right\} \subseteq\{1, \ldots, m\}$ the element $e_{A}=e_{j_{1}} e_{j_{2}}, \ldots e_{j_{r}}$, for $1 \leq j_{1}<$ $j_{2}<\ldots<j_{r} \leq m$, with $e_{\emptyset}=1$. The complexification of this real algebra is the composition $\mathbb{C}_{p, q}:=\mathbb{C} \otimes \mathbb{R}_{p, q}$ which has dimension $2^{m}$ over $\mathbb{C}$. In this article, we will consider the complex algebra $\mathbb{C}_{0, m}$ : every basis element $e_{j}$ squares up to -1 .

Clifford analysis - discrete setting. We will work in the so-called split discrete setting, introduced in [4]. Split up the basis elements $e_{j}$ of $\mathbb{C}_{m, 0}$ into forward and backward basic vectors $\boldsymbol{e}_{j}^{+}$and $\boldsymbol{e}_{j}^{-}$, such that $\boldsymbol{e}_{j}^{+}+\boldsymbol{e}_{j}^{-}=e_{j}, j=1, \ldots, m$, there are no preferential Cartesian directions (rotational invariance) and moreover, the positive and negative orientations of any cartesian direction play an equivalent role (reflection invariance). We consider the algebra over the set $\left\{\boldsymbol{e}_{j}^{+}, \boldsymbol{e}_{j}^{-} \mid j=\right.$ $1, . ., m\}$, satisfying the following relations:

$$
\begin{aligned}
& \left\{\boldsymbol{e}_{j}^{-}, \boldsymbol{e}_{k}^{-}\right\}:=\boldsymbol{e}_{j}^{-} \boldsymbol{e}_{k}^{-}+\boldsymbol{e}_{k}^{-} \boldsymbol{e}_{j}^{-}=0, \quad j, k \in\{1, \ldots, m\}, \\
& \left\{\boldsymbol{e}_{j}^{+}, \boldsymbol{e}_{k}^{+}\right\}:=\boldsymbol{e}_{j}^{+} \boldsymbol{e}_{k}^{+}+\boldsymbol{e}_{k}^{+} \boldsymbol{e}_{j}^{+}=0, \quad j, k \in\{1, \ldots, m\}, \\
& \left\{\boldsymbol{e}_{j}^{+}, \boldsymbol{e}_{k}^{-}\right\}:=\boldsymbol{e}_{j}^{+} \boldsymbol{e}_{k}^{-}+\boldsymbol{e}_{k}^{-} \boldsymbol{e}_{j}^{+}=\delta_{j k}, \quad j, k \in\{1, \ldots, m\} .
\end{aligned}
$$

Remark that these relations are consistent with the relations mentioned in (2.1). The complex field $\mathbb{C}$ can be identified with the subalgebra of scalars. The basis elements $\left\{\boldsymbol{e}_{j}^{+}, \boldsymbol{e}_{j}^{-} \mid j=1, \ldots, m\right\}$ form a basis of the space of 1-vectors. Let the wedge product (also called exterior product) be defined as follows

$$
\boldsymbol{e}_{j}^{ \pm} \wedge \boldsymbol{e}_{k}^{ \pm}:=\boldsymbol{e}_{j}^{ \pm} \boldsymbol{e}_{k}^{ \pm}-\boldsymbol{e}_{k}^{ \pm} \boldsymbol{e}_{j}^{ \pm} .
$$

Then the elements in $\left\{\boldsymbol{e}_{j}^{ \pm} \wedge \boldsymbol{e}_{k}^{ \pm} \mid j, k=1, \ldots m\right\}$ form a basis for the space of 2-vectors or bivectors. In general, $\left\{\boldsymbol{e}_{j_{1}}^{ \pm} \wedge \boldsymbol{e}_{j_{2}}^{ \pm} \wedge \ldots \wedge \boldsymbol{e}_{j_{r}}^{ \pm} \mid j_{1}, \ldots, j_{r} \in\{1, \ldots, m\}\right.$ form the basis for the $r$-vectors. 
Remark 2.1. We started from the classical Clifford algebra $\mathbb{C}_{m, 0}$ with dimension $2^{m}$ and split up the basis elements in a forward and backward vector. Because of that, the dimension raises to $\left(2^{m}\right)^{2}=2^{2 m}$. This raising can be seen as a lift from $\mathbb{C}_{m, 0}$ to $\mathbb{C}_{m, m}$ : one can compare the sum $\boldsymbol{e}_{j}^{+}+\boldsymbol{e}_{j}^{-}$to a continuous basis element $e_{j}$ with $e_{j}^{2}=1$, while the basis element $\boldsymbol{e}_{j}^{+}-\boldsymbol{e}_{j}^{-}$behaves like $e_{j}$ with $e_{j}^{2}=-1$.

Definition 2.2. A main type of involution is the Hermitian conjugation ${ }^{\dagger}$ :

$$
\left(\boldsymbol{e}_{j}^{+}\right)^{\dagger}:=\boldsymbol{e}_{j}^{-} \text {and }\left(\boldsymbol{e}_{j}^{-}\right)^{\dagger}:=\boldsymbol{e}_{j}^{+} .
$$

Hermitian conjugation switches the order of multiplication:

$$
(a b)^{\dagger}:=b^{\dagger} a^{\dagger}
$$

with $a, b \in \mathbb{C}_{m, m}$. For any $r \in \mathbb{C},(r a)^{\dagger}=\bar{r} a^{\dagger}$, where $r$ is the usual complex conjugation.

Remark 2.3. Hermitian conjugation is the discretization of hermitean conjugation in $\mathbb{C}_{m, m}$ and a generalisation of complex conjugation in $\mathbb{C}$. It holds that $\left(\boldsymbol{e}_{j}^{+}+\boldsymbol{e}_{j}^{-}\right)^{\dagger}=\boldsymbol{e}_{j}^{+}+\boldsymbol{e}_{j}^{-}$and $\left(\boldsymbol{e}_{j}^{+}-\boldsymbol{e}_{j}^{-}\right)^{\dagger}=\boldsymbol{e}_{j}^{-}-\boldsymbol{e}_{j}^{+}$.

We will from now on work with functions defined on the equidistant grid $\mathbb{Z}^{m}$, with values in the Clifford Algebra $\mathbb{C}_{m, 0}$. Classical partial derivatives are replaced by the so-called forward and backward differences for $j=1, \ldots, m$, by analogy with the classical left and right limit of differentiation:

$$
\begin{aligned}
\Delta_{j}^{+} f(x) & :=f\left(x+e_{j}\right)-f(x), \\
\Delta_{j}^{-} f(x) & :=f(x)-f\left(x-e_{j}\right),
\end{aligned}
$$

where now $x \in \mathbb{Z}^{m}$

Definition 2.4. The discrete Dirac operator is defined as

$$
\partial:=\sum_{j=1}^{m} \boldsymbol{e}_{j}^{+} \Delta_{j}^{+}+\boldsymbol{e}_{j}^{-} \Delta_{j}^{-} .
$$

This Dirac operator factorises the star Laplacian

$$
\Delta^{*}:=\sum_{j=1}^{m} \Delta_{j}^{+} \Delta_{j}^{-}=\sum_{j=1}^{m} \Delta_{j}^{-} \Delta_{j}^{+}=\partial^{2},
$$

analogeous to classical Clifford analysis where the continuous Dirac operator also factorises the Laplacian $\Delta_{x}$. On the coordinate level, we will denote $\partial_{j}=$ $\boldsymbol{e}_{j}^{+} \Delta_{j}^{+}+\boldsymbol{e}_{j}^{-} \Delta_{j}^{-}$, such that $\partial=\sum_{j=1}^{m} \partial_{j}$. 
The Dirac operator and the forward and backward differences are lowering operators: they lower the degree of a polynomial by 1 . In order to create corresponding raising operators, which we will denote by

$$
\xi=\sum_{j=1}^{m} \xi_{j}=\sum_{j=1}^{m} \boldsymbol{e}_{j}^{+} X_{j}^{-}+\boldsymbol{e}_{j}^{-} X_{j}^{+},
$$

we use the skew Weyl-relations

$$
\begin{aligned}
\Delta_{j}^{+} X_{j}^{+}-X_{j}^{-} \Delta_{j}^{-} & =1, \\
\Delta_{j}^{-} X_{j}^{-}-X_{j}^{+} \Delta_{j}^{+} & =1, \\
\partial_{j} \xi_{j}-\xi_{j} \partial_{j} & =1 .
\end{aligned}
$$

Moreover, we define the discrete Euler operator as $\mathbb{E}=\sum_{j=1}^{m} \xi_{j} \partial_{j}$, for which the same intertwining relations as in the continuous setting hold:

$$
\begin{aligned}
\partial \xi+\xi \partial & =2 \mathbb{E}+m, \\
\partial E & =E \partial+\partial, \\
E \xi & =\xi E+\xi .
\end{aligned}
$$

From the relations (2.4) and (2.5), we find how the raising operators $X_{j}^{ \pm}$and hence $\xi_{j}$ act on polynomials. In particular, $\xi_{j}^{k}[1]$, which are the basic discrete polynomials of degree $k>1$, are given by

$$
\begin{aligned}
\xi_{j}^{2 k+1}[1] & =x_{j} \prod_{s=1}^{k}\left(x_{j}^{2}-s^{2}\right)\left(\boldsymbol{e}_{j}^{+}+\boldsymbol{e}_{j}^{-}\right), \\
\xi_{j}^{2 k}[1] & =\left(x_{j}^{2}+k x_{j}\left(\boldsymbol{e}_{j}^{+} \boldsymbol{e}_{j}^{-}-\boldsymbol{e}_{j}^{-} \boldsymbol{e}_{j}^{+}\right)\right) \prod_{s=1}^{k-1}\left(x_{j}^{2}-s^{2}\right) .
\end{aligned}
$$

The roots of $\xi_{j}^{2 k+1}[1]$ are given by $-k, \ldots, k$, while those of $\xi_{j}^{2 k}[1]$ are given by $-(k-1), \ldots, k-1$.

Equations (2.8) show the action of $\xi_{j}$ from the left on discrete polynomials. It will be necessary to translate the right action to a left action, when we will work with discrete distributions. Note that, as $\xi_{j}$ are vectorial operators and since the (discrete) Clifford algebra is non commutative, the position of the basis elements $\boldsymbol{e}_{j}^{ \pm}$on the left or on the right of the function $\xi$ acts upon, can play an important role. It has been proven in [14], lemma 3.5.1 that:

$$
\begin{aligned}
& \left(\xi_{j}^{k}[1]\right) \partial_{j}^{\dagger}=\partial_{j}\left(\xi_{j}^{k}[1]\right), \\
& \left(\xi_{j}^{k}[1]\right) \xi_{j}^{\dagger}=\xi_{j}^{k+1}[1] .
\end{aligned}
$$


Definition 2.5. The discrete Euler operator is given by

$$
\mathbb{E}:=\sum_{j=1}^{m}\left(\boldsymbol{e}_{j}^{+} \boldsymbol{e}_{j}^{-} X_{j}^{-} \Delta_{j}^{-}+\boldsymbol{e}_{j}^{-} \boldsymbol{e}_{j}^{+} X_{j}^{+} \Delta_{j}^{+}\right) .
$$

A polynomial $P_{k}$ is called discrete homogeneous of degree $k$ if it is an eigenfunction of the discrete Euler operator with eigenvalue $k$ :

$$
\mathbb{E} P_{k}=k P_{k} .
$$

The basic discrete polynomials $\xi_{j}^{k}[1]$ are homogeneous of degree $k$. Moreover, they span the subvectorspace of discrete homogeneous polynomials of degree $k$. From (2.8), it is also clear that the discrete notion of homogenity does not coincide with the continuous definition. For example, a restriction of a continuous homogeneous polynomial to the grid does not give a discrete homogeneous polynomial in the discrete sense.

Every discrete function admits a unique discrete Taylor series expansion, which is a finite or infinite series of the basic homogeneous polynomials. In one dimension $(m=1)$, this Taylor series (see e.g. [15]) is given by

$$
f(x)=\sum_{k=0}^{\infty} \frac{1}{k !} \xi^{k}[1](x)\left[\partial^{k} f(u)\right]_{u=0}
$$

where $f$ is a discrete function. The Taylor series expansion $f(\xi)=\sum_{k \in \mathbb{N}} \xi^{k} c_{k}$ is the corresponding operator to this function. Letting this operator act on the identity function 1 , one obtains a function in the discrete variable $x$. By identifying a discrete function with its corresponding operator, the set of discrete functions is a right Clifford module.

Similar to classical analysis, distributions are a class of linear functionals acting on a particular space of functions.

Definition 2.6. A discrete distribution $F$ is defined as a linear functional acting on the left module of Clifford polynomials on $\mathbb{Z}^{m}$.

$$
\langle F, V\rangle:=\left\langle F,[1] V\left(\xi^{\dagger}\right)\right\rangle .
$$

Here, $V\left(\xi^{\dagger}\right)$ is the polynomial $V$ where every $\xi$ is replaced by its conjugate $\xi^{\dagger}$. The action of the operators $\Delta_{j}^{ \pm}$and $X_{j}^{ \pm}$on the distribution $F$ are defined in such a way that they are consistent with the skew Weyl relations:

$$
\begin{gathered}
\left\langle\Delta_{j}^{ \pm} F, V\right\rangle=-\left\langle F, V \Delta_{j}^{\mp}\right\rangle=-\left\langle F, \Delta_{j}^{\mp}[V]\right\rangle, \\
\left\langle X_{j}^{ \pm} F, V\right\rangle=\left\langle F, V X_{j}^{\mp}\right\rangle=\left\langle F, X_{j}^{\mp}[V]\right\rangle .
\end{gathered}
$$


For Clifford number $a, b$, we define

$$
\langle F a, b V\rangle:=b\langle F, V\rangle a .
$$

This implies in particular for the Dirac operator $\partial$ and the vector variable $\xi$ :

$$
\begin{aligned}
& \langle\partial F, V\rangle=-\left\langle F, V \partial^{\dagger}\right\rangle, \\
& \langle\xi F, V\rangle=\left\langle F, V \xi^{\dagger}\right\rangle .
\end{aligned}
$$

A special and important class of distributions are the discrete delta distributions $\boldsymbol{\delta}_{j}$, which evaluate a discrete function in the point $p \in \mathbb{Z}^{m}$ :

$$
\left\langle\boldsymbol{\delta}_{p}, f\right\rangle=f(p) .
$$

\subsection{Weierstrass transform and Hermite polynomials}

Of particular interest in classical analysis are Hermite polynomials $H_{n}$ and their corresponding functions $\psi_{n}$.

Definition 2.7. The continuous Hermite functions $\psi_{k}$ are defined as

$$
\psi_{k_{1}, \ldots, k_{m}}(\underline{x}):=H_{k_{1}, \ldots, k_{m}}(\underline{x}) e^{-|\underline{x}|^{2} / 4}
$$

where

$$
H_{k_{1}, \ldots, k_{m}}(\underline{x}) e^{-|\underline{x}|^{2} / 2}:=(-1)^{k_{1}+\ldots+k_{m}} \partial_{x_{1}}^{k_{1}} \ldots \partial_{x_{m}}^{k_{m}} e^{-|\underline{x}|^{2} / 2} .
$$

$H_{k_{1}, \ldots, k_{m}}(\underline{x})$ are the corresponding monic Hermite polynomials of degree $k$ in $\mathbb{R}^{m}$.

We used the notation $\underline{x}=\left(x_{1}, \ldots, x_{m}\right) \in \mathbb{R}^{m}$.

In one dimension, this definition reduces to

$$
\psi_{k}(x)=\left(\frac{1}{2} x-\frac{d}{d x}\right)^{k} e^{-x^{2} / 4}=H_{k}(x) e^{-x^{2} / 4},
$$

with

$$
H_{k}(x)=(-1)^{k} e^{\frac{x^{2}}{2}} \frac{d^{k}}{d x^{k}}\left(e^{-\frac{x^{2}}{2}}\right) .
$$

Definition 2.8. The Weierstrass space $\mathcal{W}$, is the weighted $L_{2}$-space with Gaussian weight function $G$ :

$$
L_{2}\left(\mathbb{R}^{m}, G\right):=\left\{f:\left.\mathbb{R}^{m} \rightarrow \mathbb{C}\left|\int_{\mathbb{R}^{m}}\right| f(\underline{x})\right|^{2} e^{-|\underline{x}|^{2} / 2} d \underline{x}<\infty\right\}
$$

with inner product

$$
\langle f, g\rangle_{\mathcal{W}}:=\frac{1}{\sqrt{2 \pi}^{m}} \int_{\mathbb{R}^{m}} f(\underline{x}) \overline{g(\underline{x})} e^{-|\underline{x}|^{2} / 2} d \underline{x} .
$$


The set of polynomials is dense in $\mathcal{W}$ and the Hermite polynomials $\left\{H_{n} \mid n \in \mathbb{N}\right\}$ form an orthogonal basis for $m=1$. It holds that $\left\langle H_{n}, H_{m}\right\rangle_{\mathcal{W}}=\delta_{m, n} n$ !. If $m>1$, higher dimensional Hermite polynomials $H_{k_{1}, \ldots, k_{m}}$ form an orthogonal basis with $\left\|H_{k_{1}, \ldots, k_{m}}\right\|^{2}=\left\langle H_{k_{1}, \ldots, k_{m}}, H_{k_{1}, \ldots, k_{m}}\right\rangle=k_{1} ! \ldots . k_{m}$ !

Definition 2.9. The Weierstrass transform of an integrable function $f$ on $\mathbb{R}$ is defined as the convolution of $f$ with the Gaussian kernel:

$$
\mathcal{W}[f](\underline{u}):=\frac{1}{\sqrt{2 \pi}^{m}} \int_{\mathbb{R}^{m}} e^{-|\underline{u}-\underline{x}|^{2} / 2} f(\underline{x}) d \underline{x} .
$$

The Weierstrass transform is a bounded operator on $\mathcal{L}_{2}\left(\mathbb{R}^{m}\right)$, with values in the Fischer space, the space of real analytic functions equipped with the inner product

$$
\langle f, g\rangle=\left.\bar{f}\left(\partial_{1}, \ldots, \partial_{m}\right) g\left(x_{1}, \ldots, x_{m}\right)\right|_{\underline{x}=\underline{0}} .
$$

A basis of this Fischer space is given by the natural powers of $u:\left\{u^{k} \mid k \in \mathbb{N}\right\}$, satisfying $\left\langle u^{k}, u^{l}\right\rangle=\delta_{k l} k$ !, and therefore, constituting an orthonormal system. This definition can be naturally extended with $z \in \mathbb{C}$ so that the result is a holomorphic function, i.e. an element of the Fock space. Whether the result of the Weierstrass transform is a real or complex function (in the Fischer space, resp. Fock space), does of course not influence its isometric property.

As an informative example, let us calculate the Weierstrass transform of the Hermite polynomials in one dimension:

$$
\begin{aligned}
\mathcal{W}\left[H_{n}\right](u) & =\frac{1}{\sqrt{2 \pi}} \int_{\mathbb{R}} e^{-(u-x)^{2} / 2}(-1)^{n} e^{x^{2} / 2} \frac{d^{n}}{d x^{n}}\left(e^{-x^{2} / 2}\right) d x \\
& =(-1)^{n} \frac{1}{\sqrt{2 \pi}} \int_{\mathbb{R}} e^{-u^{2} / 2+x u} \frac{d^{n}}{d x^{n}}\left(e^{-x^{2} / 2}\right) d x \\
& =\frac{1}{\sqrt{2 \pi}} \int_{\mathbb{R}} \frac{d^{n}}{d x^{n}}\left(e^{-u^{2} / 2+x u}\right) e^{-x^{2} / 2} d x \\
& =\frac{u^{n}}{\sqrt{2 \pi}} \int_{\mathbb{R}} e^{-(u-x)^{2} / 2} d x=u^{n} .
\end{aligned}
$$

This easy calculation shows that the Weierstrass transform of $H_{n}$ equals $u^{n}$. This proves that we deal with an isometry between the Weierstrass space and the so-called Fischer space: the space of real analytic functions equipped with the inner product (2.16).

\subsection{Discrete Gauss distribution and Hermite functions}

From now on, we will restrict our work to the one-dimensional case, for ease of calculation and notation. Yet our methods may be generalized to higher 
dimensional cases which would be a topic for future research. Higher dimensions involve more complicated calculations, mainly because of the non-commutative character of elements in the Clifford algebra.

The continuous Gauss distribution with its density function $f(x)=e^{-x^{2} / 2}$ is unavoidable in classical analysis. As it will be one of the main ingredients to define the discrete Weierstrass space, we give the definitions of the discrete analogue.

The discrete Gauss distribution $G$ is defined through its action on the discrete homogeneous polynomials, similar to the classical Gaussian distribution acting on the classical homogeneous polynomials $x^{n}$ (see [6]):

$$
\left\langle G, \xi^{n}[1]\right\rangle:= \begin{cases}\sqrt{2 \pi} & n=0, \\ 0 & n \text { odd }, \\ (n-1) ! ! \sqrt{2 \pi} & n \text { even, }\end{cases}
$$

with $(2 l-1) ! !=\frac{(2 l) !}{2^{l} l !}$ the double factorial. The dual Taylor series representation of $G$ is given by

$$
G:=\sqrt{2 \pi} \exp \left(\frac{\partial^{2}}{2}\right) \boldsymbol{\delta}_{0}
$$

and its density function is

$$
\sum_{p \in \mathbb{Z}} \sqrt{2 \pi} e^{-1} \operatorname{BesselI}(p, 1) \delta_{p}
$$

where $\operatorname{BesselI}(p, 1)$ is the modified Bessel function of the first kind and $\delta_{p}$ are the delta functions, which evaluate to 1 in $p \in \mathbb{Z}$ and 0 everywhere else. This can be obtained by expanding $\partial^{2 l} \boldsymbol{\delta}_{0}$ into discrete delta distributions.

Let $P(\xi)$ be a discrete polynomial in the discrete vector variable $\xi$. The distribution $P(\xi) G$ is well defined and can be calculated using the skew Weyl relations for distributions. Alternatively, the useful calculation rule

$$
\partial G=-\xi G
$$

enables us to write $P(\xi) G$ as a sum of derivatives of the $\boldsymbol{\delta}_{0}$ distribution. For a given polynomial $P$, defined on the grid, there exists a unique operator $P(\xi)$ such that $P(\xi)[1](n)=P(n), \forall n \in \mathbb{Z}$, hence there exists a unique distribution of the form $P(\xi) G$.

Discrete Hermite polynomials were introduced in [16] through the action of Hermite operator $H_{n}(\xi)$ on the constant function 1, i.e. $H_{n}(\xi)[1] . H_{n}(\xi)$ is, as an operator, a polynomial of degree $n$ in $\xi$ with real coefficients.

The Hermite operators are given by

$$
H_{2 l}(\xi)=\sum_{j=0}^{l} a_{2 j}^{2 l} \xi^{2 j}, \quad H_{2 l+1}(\xi)=\sum_{j=0}^{l} a_{2 j+1}^{2 l+1} \xi^{2 j+1},
$$


where

$$
a_{2 j}^{2 l}=(-1)^{j} 2^{l-j}\left(\begin{array}{l}
l \\
j
\end{array}\right) \frac{\Gamma\left(l+\frac{1}{2}\right)}{\Gamma\left(j+\frac{1}{2}\right)}, \quad a_{2 j+1}^{2 l+1}=(-1)^{j} 2^{l-j}\left(\begin{array}{l}
l \\
j
\end{array}\right) \frac{\Gamma\left(l+\frac{1}{2}+1\right)}{\Gamma\left(j+\frac{1}{2}+1\right)} .
$$

The absolute values of the coefficients $a_{2 j}^{2 l}$ and $a_{2 j+1}^{2 l+1}$ are equal to those of the coefficients of the continuous Hermite polynomials.

Even Hermite polynomials and operators have a scalar and a bivectorial part, while odd Hermite functions only have a vectorial part, as they only contain even, respectively odd powers of $\xi$.

A useful tool for calculations is Rodrigues' Formula ([14], p. 9-4):

$$
H_{2 k, m}(\xi) G=(-1)^{k} \partial^{2 k} G, \quad H_{2 k+1, m}(\xi) G=(-1)^{k+1} \partial^{2 k+1} G,
$$

which is an immediate consequence of equation (2.19)

Hermite functions are analogously defined as the product of a Hermite polynomial with $e^{-\xi^{2} / 4}$ :

$$
\psi_{n}(\xi):=H_{n}(\xi) e^{-\xi^{2} / 4}[1]
$$

Note that this is the action of the polynomial operator $H_{n}(\xi)$ on $e^{-\xi^{2} / 4}[1]$ and not the multiplication of the function $H_{n}(\xi)[1]$ with $e^{-\xi^{2} / 4}[1]$.

\section{Discrete Weierstrass transform}

\subsection{First considerations}

We want a discrete version of the Weierstrass transform, sending a discrete function (i.e. defined on the grid) to an analytic function in a Clifford valued Fock space. Therefore, we try to mimic the ideas of the classical definition. A basis for the continuous Weierstrass space, is given by the set of Hermite polynomials. In the calculations of $\mathcal{W}\left[H_{n}\right](z)=z^{n}$ (see (2.17)), the Gaussian function appears. Both the Hermite polynomials and Gaussian function are introduced in the discrete setting in the previous section. These elements will allow us to translate the Weierstrass transform to the split discrete setting.

Drawing inspiration from Rodriguez' formula (2.22), we define the discrete Weierstrass transformation of the discrete Hermite polynomials as follows:

Definition 3.1. The discrete Weierstrass transformation of the discrete Hermite polynomials are defined as

$$
\mathcal{W}\left[H_{n}\right](z):=\frac{1}{\sqrt{2 \pi}}\left\langle H_{n}(\xi) G, e^{-z^{2} / 2+\xi z}[1]\right\rangle .
$$


Proposition 3.2. $\mathcal{W}\left[H_{n}\right](z)=(-1)^{\left\lfloor\frac{n}{2}\right\rfloor} z^{n}, \forall n \in \mathbb{N}$

Proof. This is a straight forward calculation.

$$
\begin{aligned}
\left\langle H_{n}(\xi) G, e^{-z^{2} / 2+\xi z}[1]\right\rangle & =\left\langle(-1)^{\left\lceil\frac{n}{2}\right\rceil} \partial^{n} G, e^{-z^{2} / 2+\xi z}[1]\right\rangle \\
& =(-1)^{\left\lceil\frac{n}{2}\right\rceil} e^{-z^{2} / 2}\left\langle\partial^{n} G, \sum_{i=0}^{\infty} \frac{\xi^{i} z^{i}[1]}{i !}\right\rangle \\
& =(-1)^{\left\lfloor\frac{n}{2}\right\rfloor} e^{-z^{2} / 2} \sum_{i=0}^{\infty} \frac{z^{i}}{i !}\left\langle G, \xi^{i}[1]\left(\partial^{\dagger}\right)^{n}\right\rangle \\
& =(-1)^{\left\lfloor\frac{n}{2}\right\rfloor} e^{-z^{2} / 2} \sum_{i=0}^{\infty} \frac{z^{i}}{i !}\left\langle G, \partial^{n} \xi^{i}[1]\right\rangle \\
& =(-1)^{\left\lfloor\frac{n}{2}\right\rfloor} e^{-z^{2} / 2} \sum_{i=n}^{\infty} \frac{z^{i}}{i !} \frac{i !}{(i-n) !}\left\langle G, \xi^{i-n}[1]\right\rangle \\
& =(-1)^{\left\lfloor\frac{n}{2}\right\rfloor} e^{-z^{2} / 2} \sum_{j=0}^{\infty} \frac{z^{j+n}}{j !}\left\langle G, \xi^{j}[1]\right\rangle \\
& =(-1)^{\left\lfloor\frac{n}{2}\right\rfloor} e^{-z^{2} / 2} z^{n} \sum_{j=0}^{\infty} \frac{z^{2 j}}{(2 j) !} \sqrt{2 \pi} \frac{(2 j) !}{2^{j} j !} \\
& =(-1)^{\left\lfloor\frac{n}{2}\right\rfloor \sqrt{2 \pi} z^{n} .}
\end{aligned}
$$

This proves the proposition.

These transformations correspond to the continuous case, taking into account that the discrete Hermite polynomials are formally equal to their continuous counterparts, up to signs.

This definition can be naturally extended to any finite linear combination of Hermite polynomials:

$$
f=\sum_{n \in \mathbb{N}} H_{n} c_{n} \Rightarrow \mathcal{W}[f](z)=\sum_{n \in \mathbb{N}} \mathcal{W}\left[H_{n}\right](z) c_{n}, \quad c_{n} \in \mathbb{C}_{m, m}
$$

In the next section, we want to extend this definition for general discrete functions and find a condition which has to be fulfilled in order that the function has a Weierstrass transform.

\subsection{Discrete Weierstrass space}

The classical Weierstrass transform acts on the $\mathcal{L}_{2}\left(\mathbb{R}^{m}, G\right)$-space with results in the Fock space. In the previous section, we established a discrete version 
of this transform, only for any finite linear combination of discrete Hermite polynomials. Can we find an appropriate space of functions, similar to the classical weighted $L_{2}$-space, such that the discrete Weierstrass transform is extendable to this space?

The elementary principles of the continuous setting will lead to the definition of a discrete Weierstrass space $\mathcal{W}$. The set of discrete polynomials should be a dense subset of $\mathcal{W}$ and a basis will be formed by the Hermite polynomials. The inner product will be defined using the discrete Gauss distribution.

The set of discrete functions forms a right Clifford module in which the right submodule of polynomials is dense, by writing a discrete function $f$ in its Taylor series $f(\xi)[1]$, with $f(\xi)=\sum_{k \in \mathbb{N}} \xi^{k} c_{k}$ the corresponding operator.

Remark that the set of discrete Hermite polynomials in $\xi$ of degree less or equal to $n$, as its classical counterpart, is a set of $n+1$ linear independent polynomials. Hence, the space of discrete polynomials of degree $\leq n$ is spanned by these Hermite polynomials, i.e. every discrete polynomial in $\xi$ can be written as a finite linear combination of Hermite polynomials.

Define a sesquilinear form as follows:

$$
(f, g)=(f(\xi)[1], g(\xi)[1]):=\left\langle f(\xi) G,[1](g(\xi))^{\dagger}\right\rangle
$$

We will prove that this form defines an inner product on the space spanned by the discrete Hermite polynomials, in which the latter will form an orthogonal basis.

Remark that

$$
(f(\xi)[1])^{\dagger}=[1] \sum_{k \in \mathbb{N}} c_{k}^{\dagger}\left(\xi^{\dagger}\right)^{k}=\sum_{k \in \mathbb{N}} c_{k}^{\dagger} \xi^{k}[1] .
$$

In particular, if $f$ is a real function (i.e. every $c_{k} \in \mathbb{R}$ ) then $[1](f(\xi))^{\dagger}=f(\xi)[1]$.

Lemma 3.3. The discrete Hermite polynomials are orthogonal with respect to the inner product (3.2)

Proof. We calculate $\left(H_{n}, H_{m}\right)$. If $n \neq m$, it follows from [16] that $\left(H_{n}, H_{m}\right)=0$. Suppose $m=n=2 k$ is even.

$$
\begin{aligned}
\left(H_{2 k}, H_{2 k}\right) & =\left\langle\sum_{j=0}^{k} a_{2 j}^{2 k} \xi^{2 j} G,[1]\left(\sum_{i=0}^{k} a_{2 i}^{2 k} \xi^{2 i}\right)^{\dagger}\right\rangle=\left\langle\sum_{j=0}^{k} a_{2 j}^{2 k} \xi^{2 j} G, \sum_{i=0}^{k} a_{2 i}^{2 k}[1]\left(\xi^{2 i}\right)^{\dagger}\right\rangle \\
& =\sum_{i, j=0}^{k} a_{2 j}^{2 k} a_{2 i}^{2 k}\left\langle G, \xi^{2 i+2 j}[1]\right\rangle=\sum_{i, j=0}^{k} a_{2 j}^{2 k} a_{2 i}^{2 k} \sqrt{2 \pi} \frac{(2 i+2 j) !}{2^{i+j}(i+j) !} \\
& =\sqrt{2 \pi}(2 k) !=: \eta_{2 k} .
\end{aligned}
$$


While for $m=n=2 k+1$, we have similarly that

$$
\begin{aligned}
\left(H_{2 k+1}, H_{2 k+1}\right) & =\sum_{i, j \in \mathbb{N}} a_{2 j+1}^{2 k+1} a_{2 i+1}^{2 k+1} \sqrt{2 \pi} \frac{(2 i+2 j+2) !}{2^{i+j+1}(i+j+1) !} \\
& =\sqrt{2 \pi}(2 k+1) !=: \eta_{2 k+1} .
\end{aligned}
$$

The last step was calculated by filling in the explicit values of the coefficients $a_{j}^{k}$.

In conclusion, for $n, m \in \mathbb{N}:\left(H_{n}, H_{m}\right)=\sqrt{2 \pi} n ! \delta_{n, m}=: \eta_{n}$.

Remark 3.4. The results are the same as the inner products of Hermite polynomials in the continuous setting.

Lemma 3.5. The bilinear form (3.4) is conjugate symmetric with respect to $\dagger$.

Proof. Let $f, g$ be two discrete Clifford-valued polynomials and write them in their Hermite polynomial expansion, i.e. $f=\sum_{k \in \mathbb{N}} H_{k} a_{k}$ and $g=\sum_{l \in \mathbb{N}} H_{l} b_{l}$. We than have

$$
\begin{aligned}
(f, g) & =\left\langle f(\xi) G,[1](g(\xi))^{\dagger}\right\rangle \\
& =\sum_{k, l \in \mathbb{N}}\left\langle H_{k}(\xi) a_{k} G,[1]\left\langle H_{l}(\xi) b_{l}\right)^{\dagger}\right\rangle \\
& =\sum_{k, l \in \mathbb{N}} b_{l}^{\dagger}\left\langle H_{k}(\xi) G,[1] H_{l}\left(\xi^{\dagger}\right)\right\rangle a_{k} \\
& =\sum_{n \in \mathbb{N}} b_{n}^{\dagger} \eta_{n} a_{n},
\end{aligned}
$$

Since $G$ is scalar, the second step $a_{k} G=G a_{k}$ is allowed. Also, as $\eta_{n} \in \mathbb{R}$,

$$
(g, f)^{\dagger}=\sum_{n \in \mathbb{N}}\left(a_{n}^{\dagger} \eta_{n} b_{n}\right)^{\dagger}=\sum_{n \in \mathbb{N}} b_{n}^{\dagger} \eta_{n} a_{n}
$$

Using the orthogonality of the Hermite polynomials $H_{k}=H_{k}(\xi)[1]$, we can proof that the bilinear form in definition 3.2 is not indefinite. As the form is Clifford-valued, positive definiteness does not apply.

Remark 3.6. It is possible to take the scalar part of the right hand side of definion (3.2) in order to obtain a positive definite inner product. In that case however, one obtains a complex Hilbert space, in which the discrete Hermite polynomials are no longer basis elements.

Lemma 3.7. For a discrete function $f$, it holds that if $(f, f)$, then $f=0$. 
Proof. Let us first calculate $r^{\dagger} r$, where $r$ is an arbitrary clifford element $r=$ $a+b \boldsymbol{e}^{+}+c \boldsymbol{e}^{-}+d\left(\boldsymbol{e}^{+} \wedge \boldsymbol{e}^{-}\right)$, where $a, b, c, d$ are real or complex numbers.

$$
\begin{aligned}
&\left(a+b \boldsymbol{e}^{+}+c \boldsymbol{e}^{-}+d\left(\boldsymbol{e}^{+} \wedge \boldsymbol{e}^{-}\right)\right)^{\dagger}\left(a+b \boldsymbol{e}^{+}+c \boldsymbol{e}^{-}+d\left(\boldsymbol{e}^{+} \wedge \boldsymbol{e}^{-}\right)\right) \\
&=\left(a+b \boldsymbol{e}^{-}+c \boldsymbol{e}^{+}+d\left(\boldsymbol{e}^{+} \wedge \boldsymbol{e}^{-}\right)\right)\left(a+b \boldsymbol{e}^{+}+c \boldsymbol{e}^{-}+d\left(\boldsymbol{e}^{+} \wedge \boldsymbol{e}^{-}\right)\right) \\
&=a^{2}+a b \boldsymbol{e}^{+}+a c \boldsymbol{e}^{-}+a d\left(\boldsymbol{e}^{+} \wedge \boldsymbol{e}^{-}\right)+a b \boldsymbol{e}^{-}+b^{2} \boldsymbol{e}^{-} \boldsymbol{e}^{+}+b d \boldsymbol{e}^{-}\left(\boldsymbol{e}^{+} \wedge \boldsymbol{e}^{-}\right)+ \\
& \quad a c \boldsymbol{e}^{+}+c^{2} \boldsymbol{e}^{+} \boldsymbol{e}^{-}+c d \boldsymbol{e}^{+}\left(\boldsymbol{e}^{+} \wedge \boldsymbol{e}^{-}\right)+a d\left(\boldsymbol{e}^{+} \wedge \boldsymbol{e}^{-}\right)+b d\left(\boldsymbol{e}^{+} \wedge \boldsymbol{e}^{-}\right) \boldsymbol{e}^{+} \\
& \quad+c d\left(\boldsymbol{e}^{+} \wedge \boldsymbol{e}^{-}\right) \boldsymbol{e}^{-}+d^{2}\left(\boldsymbol{e}^{+} \wedge \boldsymbol{e}^{-}\right)^{2} \\
&=a^{2}+d^{2}+\frac{b^{2}+c^{2}}{2}+(a b+a c-c d+b d) \boldsymbol{e}^{+}+(a b+a c-c d+b d) \boldsymbol{e}^{-} \\
& \quad+\frac{c^{2}-b^{2}+4 a d}{2}\left(\boldsymbol{e}^{+} \wedge \boldsymbol{e}^{-}\right)
\end{aligned}
$$

We invoked the relations (2.2), (2.3) and

$$
\begin{aligned}
e^{ \pm}\left(e^{+} \wedge e^{-}\right) & =\mp e^{ \pm}, \quad\left(e^{+} \wedge e^{-}\right) e^{ \pm}= \pm e^{ \pm} \\
\left(e^{+} \wedge e^{-}\right)^{2} & =1, \\
\left(e^{+} \wedge e^{-}\right)^{\dagger} & =e^{+} \wedge e^{-}
\end{aligned}
$$

Let $f$ be a Clifford-valued polynomial, written as a finite linear combination of Hermite polynomials: $f=\sum_{k \in \mathbb{N}} H_{k} c_{k}$. Then

$$
(f, f)=\sum_{k, l} c_{l}^{\dagger}\left(H_{k}, H_{l}\right) c_{k}=\sum_{k \in \mathbb{N}} \eta_{k} c_{k}^{\dagger} c_{k} .
$$

It follows that if $(f, f)=0$ then $f=0$.

Proven all of the defining properties of an inner product, we have come to the next definition:

Definition 3.8 (Inner product). The inner product of two discrete functions is given by

$$
(f, g)=(f(\xi)[1], g(\xi)[1]):=\left\langle f(\xi) G,[1](g(\xi))^{\dagger}\right\rangle,
$$

with $f(\xi)$ and $g(\xi)$ the Taylor series expansions of $f$ and $g$.

The scalar part of a Clifford number is used to define the norm:

Definition 3.9. The norm of a Clifford number $a$ is defined as:

$$
\|a\|:=[a]_{0} .
$$

This naturally leads to the definition of norm of a function:

Definition 3.10. The scalar part of $(f, f)$ is defined as the norm of the discrete function $f$ :

$$
\|f\|:=[(f, f)]_{0} .
$$


By Lemma 3.7 and equation (3.3), this definition is well-defined. Remark that this definition can be naturally applied to the norm of a general Clifford-element.

Based on the findings in this section, we define the discrete Weierstrass space as follows:

Definition 3.11. The discrete Weierstrass space $\mathcal{W}$ is the completion of the right Clifford module of Hermite polynomials in $\xi$ in the norm (3.4): $f \in$ $\mathcal{W} \Leftrightarrow f=\sum_{n \in \mathbb{N}}^{\infty} H_{n} a_{n}$ with $(f, f)<\infty$.

Remark that this condition can be rewritten as $\left(\frac{\left[a_{n}\right]_{0}}{\sqrt{\eta_{n}}}\right)_{n} \in \ell_{2}$, where $\ell_{2}$ is defined in the usual way as the space of sequences $\left(x_{n}\right)_{n}$ satisfying

$$
\sum_{n \in \mathbb{N}}\left|x_{n}\right|^{2}<\infty
$$

With this newly introduced Weierstrass space, we can expand the definition of the discrete Weierstrass transform to all functions in $\mathcal{W}$, as they are convergent (for the $\mathcal{W}$-inner product) series of Hermite polynomials.

Definition 3.12 (Discrete Weierstrass transform). For a discrete function $f \in$ $\mathcal{W}, f=\sum_{k \in \mathbb{N}}^{\infty} H_{k} a_{k}$, its Weierstrass transform is defined as

$$
\mathcal{W}[f](z)=\frac{1}{\sqrt{2 \pi}}\left\langle f(\xi) G, e^{-z^{2} / 2+\xi z}[1]\right\rangle=\sum_{n \in \mathbb{N}} \mathcal{W}\left[H_{n}\right] a_{n}=\sum_{n \in \mathbb{N}}(-1)^{\frac{\lfloor n\rfloor}{2}} a_{n} z^{n}
$$

The distribution $f(\xi) G$ is calculated by writing $f$ in its Taylor series expansion, hence a (possible infinite) series of polynomials in the operator $\xi$.

If $f=\sum_{n \in \mathbb{N}}^{\infty} H_{n} a_{n} \in \mathcal{W}$, then $\mathcal{W}[f]=\sum_{n \in \mathbb{N}} z^{n} a_{n}$, and

$$
\begin{aligned}
\langle\mathcal{W}[f], \mathcal{W}[f]\rangle & =\frac{1}{\sqrt{2 \pi}} \sum_{n \in \mathbb{N}}\left\langle z^{n} a_{n}, \sum_{m \in \mathbb{N}} z^{m} a_{m}\right\rangle \\
& =\frac{1}{\sqrt{2 \pi}} \sum_{m, n \in \mathbb{N}} a_{m} a_{n}\left\langle z^{n}, z^{m}\right\rangle \\
& =\frac{1}{\sqrt{2 \pi}} \sum_{n \in \mathbb{N}}\left|a_{n}\right|^{2} n ! .
\end{aligned}
$$

As $\left(\frac{a_{n}}{\sqrt{\eta_{n}}}\right)_{n} \in \ell_{2}$, this series converges in the Fock space.

Of course any polynomial is an element of $\mathcal{W}$. What other elements can we distinguish? Let us have a look at some examples. 


\subsection{Examples}

Example 1. For any linear combination of Hermite polynomials, one clearly has

$$
\sum_{k, l=0}^{\infty}\left(H_{k} c_{k}, H_{l} b_{l}\right)=\sum_{k=0}^{\infty} b_{k}^{\dagger}\left(H_{k}, H_{k}\right) c_{k}=\sum_{k=0}^{\infty} b_{k}^{\dagger} \eta_{k} c_{k} .
$$

Example 2. The inner product of two basic discrete polynomials follows immediately from the definition of the Gaussian $G$.

$$
\begin{aligned}
& \left(\xi^{k}[1], \xi^{l}[1]\right)=\left\langle\xi^{k} G,[1]\left(\xi^{\dagger}\right)^{l}\right\rangle \\
& =\left\langle G,[1]\left(\xi^{\dagger}\right)^{l}\left(\xi^{\dagger}\right)^{k}\right\rangle \\
& =\left\langle G, \xi^{k+l}[1]\right\rangle= \begin{cases}\sqrt{2 \pi} & k+l=0, \\
\sqrt{2 \pi}(k+l-1) ! ! & k+l \text { even, } \\
0 & k+l \text { odd } .\end{cases}
\end{aligned}
$$

Example 3. We calculate the $\left(e^{\lambda \xi}, e^{\lambda \xi}\right), \lambda \in \mathbb{C}$.

$$
\begin{aligned}
\left(e^{\lambda \xi}, e^{\lambda \xi}\right) & =\left\langle e^{\lambda \xi} G,[1] e^{\lambda \xi^{\dagger}}\right\rangle=\left\langle G, e^{\lambda \xi}[1] e^{\lambda \xi^{\dagger}}[1]\right\rangle=\left\langle G, e^{2 \lambda \xi}[1]\right\rangle \\
& =\sum_{s=0}^{\infty} \frac{(2 \lambda)^{s}}{s !}\left\langle G, \xi^{s}[1]\right\rangle=\sum_{\ell=0}^{\infty} \frac{(2 \lambda)^{2 \ell}}{(2 \ell) !}\left\langle G, \xi^{2 \ell}[1]\right\rangle=\sum_{\ell=0}^{\infty} \frac{(2 \lambda)^{2 \ell}}{(2 \ell) !} \sqrt{2 \pi} \frac{(2 \ell) !}{2^{\ell} \ell !} \\
& =\sum_{\ell=0}^{\infty} \sqrt{2 \pi} \frac{\lambda^{2 \ell} 2^{\ell}}{\ell !}=\sqrt{2 \pi} e^{2 \lambda^{2}}
\end{aligned}
$$

Hence for every $\lambda \in \mathbb{C}, e^{\lambda \xi}$ is an element of $\mathcal{W}$.

Example 4. Not every discrete exponential function is an element of $\mathcal{W}$ :

$$
\begin{aligned}
\left(e^{\lambda \xi^{2}}[1], e^{\lambda \xi^{2}}[1]\right) & =\left\langle G, e^{2 \lambda \xi^{2}}[1]\right\rangle=\sum_{s=0}^{\infty} \frac{(2 \lambda)^{s}}{s !}\left\langle G, \xi^{2 s}[1]\right\rangle \\
& =\sum_{s=0}^{\infty} \frac{(2 \lambda)^{s}}{s !} \sqrt{2 \pi} \frac{(2 s) !}{2^{s} s !}=\sum_{s=0}^{\infty} \sqrt{2 \pi} \lambda^{s}\left(\begin{array}{c}
2 s \\
s
\end{array}\right) .
\end{aligned}
$$

The convergence of this series depends on the parameter $\lambda$. Denoting the general term by $a_{s}$, this series is convergent by d'Alemberts criterium, if

$$
\lim _{s \rightarrow \infty}\left|\frac{a_{s+1}}{a_{s}}\right|<1 \Leftrightarrow|\lambda|<\frac{1}{4} .
$$


The cases for $\lambda= \pm \frac{1}{4}$ are the borderline: after some tedious calculations, the series with $\lambda=-\frac{1}{4}$ is also convergent with value $\sqrt{\pi}$, while the positive value $\frac{1}{4}$ is divergent.

Other examples are

$$
\begin{array}{cl}
\left(e^{-\xi^{2} / 8}, e^{-\xi^{2} / 8}\right)=\frac{2 \sqrt{3}}{3} \sqrt{\pi}, & \left(e^{-\xi^{2} / 6}, e^{-\xi^{2} / 6}\right)=\frac{\sqrt{30}}{5} \sqrt{\pi}, \\
\left(e^{\xi^{2} / 8}, e^{\xi^{2} / 8}\right)=2 \sqrt{\pi}, \quad\left(e^{\xi^{2} / 6}, e^{\xi^{2} / 6}\right)=\sqrt{6 \pi}
\end{array}
$$

The inner product $\left(e^{ \pm \xi^{2} / 2}, e^{ \pm \xi^{2} / 2}\right)$ is divergent. This example shows that it is indeed useful to put a condition on the set of functions in $\mathcal{W}$. Remark that in the classical setting however, the continuous Gaussian $\exp \left(-x^{2} / 2\right)$ is an element of the Weierstrass space. The difference is found in the fact that our weight function is the Gaussian distribution, defined with $\partial$ instead of $\xi$, while the discrete function $e^{-\xi^{2} / 2}[1]$ will tend to $\infty$ for $x \rightarrow \infty$.

Example 5. The inner product of two basic vectors is:

$$
\begin{aligned}
& \left(e^{+}, e^{+}\right)=\left\langle e^{+} G,[1] e^{-}\right\rangle=\left\langle G,[1] e^{-} e^{+}\right\rangle=\left\langle G, e^{-} e^{+}[1]\right\rangle=\sqrt{2 \pi} e^{-} e^{+}=\frac{\sqrt{2 \pi}}{2} \\
& \left(e^{-}, e^{-}\right)=\frac{\sqrt{2 \pi}}{2} \\
& \left(e^{+}, e^{-}\right)=\left\langle e^{+} G,[1] e^{+}\right\rangle=\left\langle G, e^{+} e^{+}[1]\right\rangle=0
\end{aligned}
$$

We can interpret these results as orthogonality relations of the basic elements $e^{+}$and $e^{-}$. As a result, $\left(e^{+} \pm e^{-}, e^{+} \pm e^{-}\right)=+\sqrt{2 \pi}$

Let us now calculate the Weierstrass transform of the previous examples.

1. We start with the basic discrete homogeneous polynomials of even degree

$$
\begin{aligned}
\mathcal{W}\left[\xi^{2 k}[1]\right](z) & =\frac{1}{\sqrt{2 \pi}}\left\langle\xi^{2 k} G, e^{-z^{2} / 2+\xi z}[1]\right\rangle \\
& =\frac{1}{\sqrt{2 \pi}} e^{-z^{2} / 2}\left\langle\xi^{2 k} G, \sum_{l=0}^{\infty} \frac{\xi^{l} z^{l}}{l !}[1]\right\rangle \\
& =\frac{1}{\sqrt{2 \pi}} e^{-z^{2} / 2} \sum_{l=0}^{\infty} \frac{z^{l}}{l !}\left\langle G, \xi^{l+2 k}[1]\right\rangle \\
& =e^{-z^{2} / 2} \sum_{l=0}^{\infty} \frac{z^{2 l}}{(2 l) !}\left\langle G, \xi^{2 l+2 k}[1]\right\rangle \\
& =e^{-z^{2} / 2} \sum_{l=0}^{\infty} \frac{z^{2 l}}{(2 l) !} \frac{(2 l+2 k) !}{2^{l+k}(l+k) !} \\
& =e^{-z^{2} / 2}(2 k-1) ! !{ }_{1} F_{1}\left(k+\frac{1}{2} ; \frac{1}{2} ; \frac{z^{2}}{2}\right) .
\end{aligned}
$$


For odd powers of $\xi$, we find

$$
\begin{aligned}
\mathcal{W}\left[\xi^{2 k+1}[1]\right](z) & =e^{-z^{2} / 2} \sum_{l=0}^{\infty} \frac{z^{2 l+1}}{(2 l+1) !}\left\langle G, \xi^{2 l+2 k+2}[1]\right\rangle \\
& =e^{-z^{2} / 2} \sum_{l=0}^{\infty} \frac{z^{2 l+1}}{(2 l+1) !} \frac{(2 l+2 k+2) !}{2^{l+k+1}(l+k+1) !} \\
& =e^{-z^{2} / 2} z(2 k+1) ! !_{1} F_{1}\left(k+\frac{3}{2} ; \frac{3}{2} ; \frac{z^{2}}{2}\right) .
\end{aligned}
$$

${ }_{1} F_{1}(a ; b ; z)$ is the generalized hypergeometric function, converging for all finite values of $z$. Substituting different values for $k$, we see the following concrete results:

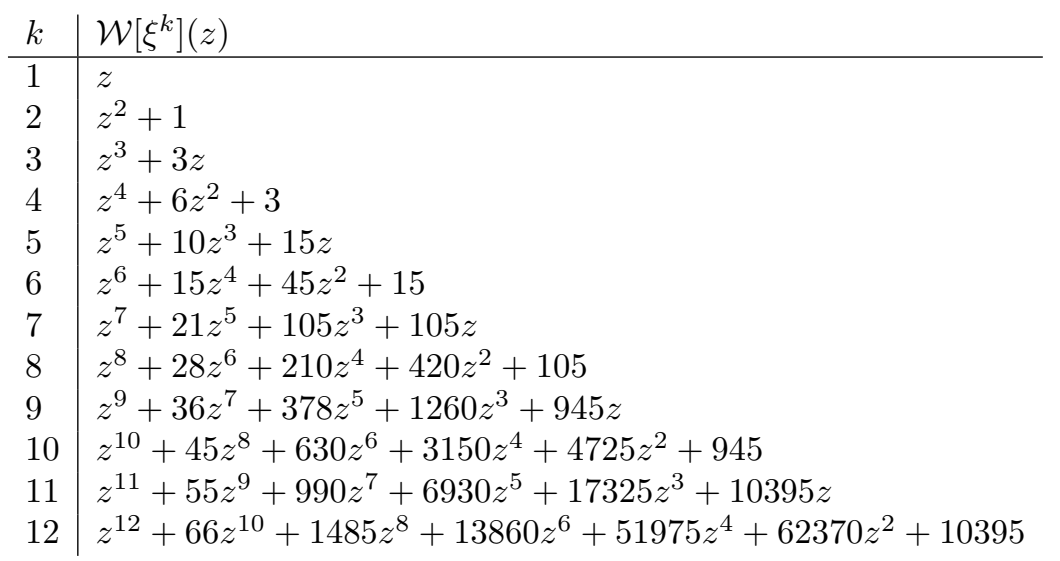

In these polynomials, we recognize the same coefficients as in the (continuous) Hermite polynomials, up to sign. More specific, we see that

$$
\begin{aligned}
\mathcal{W}\left[\xi^{2 k}\right](z) & =(-1)^{k} H_{2 k}(i z), \\
\mathcal{W}\left[\xi^{2 k+1}\right](z) & =(-1)^{k+1} i H_{2 k+1}(i z) .
\end{aligned}
$$

A natural question is to compare these results with the classical (continuous) case. Indeed, the continuous Weierstrass transform of the (continuous) polynomials $x^{k}$ are the same polynomials as in the table above. 
2. Exponential functions:

$$
\begin{aligned}
\mathcal{W}\left[e^{\lambda \xi}\right](z) & =\left\langle e^{\lambda \xi} G, e^{-z^{2} / 2+\xi z}[1]\right\rangle \\
& =\sum_{l=0}^{\infty} \frac{1}{l !} \lambda^{l}\left\langle\xi^{l} G, e^{-z^{2} / 2+\xi z}[1]\right\rangle \\
& =\sum_{l=0}^{\infty} \frac{\lambda^{l}}{l !} \mathcal{W}\left[\xi^{l}\right](z) \\
& =\sum_{l=0}^{\infty} \frac{\lambda^{2 l}}{(2 l) !}(-1)^{l} H_{2 l}(i z)+\frac{\lambda^{2 l+1}}{(2 l+1) !}(-1)^{l+1} i H_{2 l+1}(i z) .
\end{aligned}
$$

To calculate this sum of Hermite polynomials, we use the formulas ([17])

$$
\begin{gathered}
\sum_{k=0}^{\infty} \frac{2^{k} H_{2 k}(\sqrt{2} x) \omega^{k}}{(2 n) !}=e^{-\omega} \cos (2 x \sqrt{-\omega}), \\
\sum_{k=0}^{\infty} \frac{2^{k+\frac{1}{2}} H_{2 k+1}(\sqrt{2} x) \omega^{k}}{(2 n+1) !}=\frac{e^{-\omega}}{\sqrt{-\omega}} \sin (2 x \sqrt{-\omega}) .
\end{gathered}
$$

Making the right substitutions and letting $\omega=\frac{-\lambda^{2}}{2}$, we arrive at

$$
\begin{aligned}
\mathcal{W}\left[e^{\lambda \xi}\right](z) & =e^{-\omega} \cos (\sqrt{2} i z \sqrt{-\omega})+\frac{\lambda i}{\sqrt{-\omega}} e^{-\omega} \sin (\sqrt{2} i z \sqrt{-\omega}) \\
& =e^{\lambda^{2} / 2} \cos \left(\sqrt{2} i z \sqrt{\frac{\lambda^{2}}{2}}\right)-\frac{\lambda i}{\sqrt{\frac{\lambda^{2}}{2}}} \frac{e^{\lambda^{2} / 2}}{\sqrt{2}} \sin \left(\sqrt{2} i z \sqrt{\frac{\lambda^{2}}{2}}\right) \\
& =e^{\lambda^{2} / 2}[\cos (i \lambda z)+i \sin (i \lambda z)] \\
& =e^{\lambda^{2} / 2+i \lambda z} .
\end{aligned}
$$

3. Let $|\lambda|<\frac{1}{4}$, such that $e^{\lambda \xi^{2}} \in \mathcal{W}$.

$$
\begin{aligned}
\mathcal{W}\left[e^{\lambda \xi^{2}}\right](z) & =\left\langle e^{\lambda \xi^{2}} G, e^{-z^{2} / 2+\xi z}[1]\right\rangle \\
& =\sum_{l=0}^{\infty} \frac{1}{l !} \lambda^{l}\left\langle\xi^{2 l} G, e^{-z^{2} / 2+\xi z}[1]\right\rangle \\
& =\sum_{l=0}^{\infty} \frac{\lambda^{l}}{l !} \mathcal{W}\left[\xi^{2 l}\right](z) \\
& =\sum_{l=0}^{\infty} \frac{\lambda^{l}}{l !}(-1)^{l} H_{2 l}(i z) .
\end{aligned}
$$

Adding both formulas in (3.7) and keeping in mind that $H_{n}(-z)=(-1)^{n} H_{n}(z)$, we find

$$
\sum_{n=0}^{\infty} \frac{H_{n}(z) \omega^{n}}{\left\lfloor\frac{n}{2}\right\rfloor !}=\left(1+2 z \omega+4 \omega^{2}\right)\left(1+4 \omega^{2}\right)^{-3 / 2} e^{\frac{4 \omega^{2} z^{2}}{1+4 \omega^{2}}} .
$$


Substituting this result in the calculation of $\mathcal{W}\left[e^{\lambda \xi^{2}}\right](z)$, with $\omega=\frac{-\lambda^{2}}{2}$,

$$
\mathcal{W}\left[e^{\lambda \xi^{2}}\right](z)=e^{\frac{4 \lambda z^{2}}{1-4 \lambda}}(1-4 \lambda)^{-\frac{1}{2}} .
$$

\section{Discrete $\delta$-functions}

As they are the building blocks of discrete function theory, we investigate the

discrete $\delta$-functions and check if they are elements of $\mathcal{W}$. Let $\delta_{0}=\delta_{0}(\xi)[1]$ be the $\delta_{0}$-function which takes values 0 everywhere except in the origin where it is 1. Let $\delta_{0}(\xi)=\sum_{l \in \mathbb{Z}} \xi^{\ell} c_{\ell}$ the corresponding Taylor series operator. We search for an expression $\delta_{0}=\delta_{0}(\xi)[1]=\sum_{n \in \mathbb{N}} H_{n}(\xi) d_{n}[1]$. It holds that $\delta_{0}(\xi)[1]=$ $\delta_{0}=\delta_{0}^{\dagger}=\left(\delta_{0}(\xi)[1]\right)^{\dagger}$, because $\delta_{0}$ is real. If the coefficients $d_{n}$ exist, they must satisfy the relations

$$
\begin{aligned}
\left(H_{n}, \delta_{0}\right) & =\left\langle H_{n}(\xi) G,[1] \sum_{\ell \in \mathbb{N}} d_{\ell}^{\dagger} H_{\ell}(\xi)^{\dagger}\right\rangle=\left\langle H_{n}(\xi) G, \sum_{\ell \in \mathbb{N}} d_{\ell}^{\dagger} H_{\ell}(\xi)[1]\right\rangle \\
& =d_{n}^{\dagger}\left\langle H_{n}(\xi) G, H_{n}\right\rangle=d_{n}^{\dagger} \eta_{n} .
\end{aligned}
$$

On the one hand, we have

$$
\left(H_{n}, \delta_{0}\right)=\left\langle H_{n}(\xi) G, \delta_{0}\right\rangle=\sum_{s \in \mathbb{Z}}\left(H_{n}(\xi) G\right)(s) \delta_{0}(s),
$$

because the distribution $H_{k}(\xi) G$ corresponds to a function on $\mathbb{Z}$, where the action is pointwise. In this way, we can use $\delta_{0}$ as a density function on $\mathbb{Z}$. So $\left(H_{n}, \delta_{0}\right)=\left(H_{n}(\xi) G\right)(0)$. On the other hand, one can also consider the Taylor series $\delta_{0}(\xi)=\sum_{l \in \mathbb{N}} \xi^{\ell} c_{\ell}$ and substitute it into the inner product $\left(H_{n}, \delta_{0}\right)$ :

$$
\left(H_{n}, \delta_{0}\right)=\left\langle H_{n}(\xi) G,[1] \sum_{\ell \in \mathbb{N}} c_{\ell}^{\dagger} \xi^{\ell}\right\rangle=\sum_{l \in \mathbb{N}} c_{\ell}^{\dagger}\left\langle H_{n} G,[1] \xi^{\ell}\right\rangle,
$$

which should equal $d_{n}^{\dagger} \eta_{n}$. Remark that this gives a finite sum, as for $n>\ell$, the result $\left\langle H_{n} G, \xi^{\ell}\right\rangle$ is zero. This gives us two ways to calculate the coefficients $d_{n}$.

$$
d_{n}^{\dagger}=\frac{\left(H_{n}, \delta_{0}\right)}{\eta_{n}} .
$$

\subsection{Taylor series method}

Expanding $\delta_{0}$ by its Taylor series, we have (see [15])

$$
\delta_{0}(x)=\sum_{l=0}^{\infty} \frac{(-1)^{\ell}}{l ! l !} \xi^{2 l}[1](x)+\sum_{l=0}^{\infty} \frac{(-1)^{l+1}}{(l+1) ! l !} \xi^{2 l+1}[1](x)\left(\boldsymbol{e}^{+}-\boldsymbol{e}^{-}\right) .
$$


As $\left\langle G, \xi^{s}[1]\right\rangle=0$ for $s$ odd, only the first part of the Taylor series is of importance in order to calculate $\left(H_{n}, \delta_{0}\right)$. Using Rodriguez formula $(2.22)$, we get

$$
\begin{aligned}
\left\langle H_{2 k}(\xi) G,[1] \delta_{0}^{\dagger}\right\rangle & =\sum_{l=0}^{\infty} \frac{(-1)^{\ell}}{l ! l !}\left\langle(-1)^{k} \partial^{2 k} G, \xi^{2 l}[1]\right\rangle \\
& =\sum_{l=0}^{\infty} \frac{(-1)^{l+k}}{l ! l !}\left\langle G, \partial^{2 k} \xi^{2 l}[1]\right\rangle \\
& =\sum_{l=k}^{\infty} \frac{(-1)^{l+k}}{l ! l !} \frac{(2 l) !}{(2 l-2 k) !}\left\langle G, \xi^{2 l-2 k}[1]\right\rangle \\
& =\sum_{l=k}^{\infty} \frac{(-1)^{l+k}}{l ! l !} \frac{(2 l) !}{(2 l-2 k) !} \sqrt{2 \pi} \frac{(2 l-2 k) !}{2^{l-k}(l-k) !} \\
& =\sum_{l=k}^{\infty}(-1)^{l+k} \frac{\sqrt{2 \pi}}{l ! l !} \frac{(2 l) !}{2^{l-k}(l-k) !} \\
& =\sqrt{2 \pi}\left(\begin{array}{c}
2 k \\
k
\end{array}\right){ }_{1} F_{1}\left(k+\frac{1}{2}, k+1,-2\right) .
\end{aligned}
$$

We calculate $\left(H_{2 k+1}, \delta_{0}\right)$. Analoguously as in the case above, only the second part of the Taylor series is of importance.

$$
\begin{aligned}
\left\langle H_{2 k+1}(\xi) G,[1] \delta_{0}^{\dagger}\right\rangle & =\sum_{l=0}^{\infty} \frac{(-1)^{l+1}}{(l+1) ! l !}\left(e^{-}-e^{+}\right)\left\langle H_{2 k+1}(\xi) G,[1]\left(\xi^{2 l+1}\right)^{\dagger}\right\rangle \\
& =\sum_{l=0}^{\infty} \frac{(-1)^{l+1}}{(l+1) ! l !}\left(e^{-}-e^{+}\right)\left\langle(-1)^{k+1} \partial^{2 k+1} G, \xi^{2 l+1}[1]\right\rangle \\
& =\sum_{l=0}^{\infty} \frac{(-1)^{l+k+1}}{(l+1) ! l !}\left(e^{-}-e^{+}\right)\left\langle G, \xi^{2 l+1}[1]\left(\partial^{2 k+1}\right)^{\dagger}\right\rangle \\
& =\sum_{l=k}^{\infty} \frac{(-1)^{l+k+1}}{(l+1) ! l !} \frac{(2 l+1) !}{(2 l-2 k) !}\left(e^{-}-e^{+}\right)\left\langle G, \xi^{2 l-2 k}[1]\right\rangle \\
& =\sum_{l=k}^{\infty} \frac{(-1)^{l+k+1}}{(l+1) ! l !} \frac{(2 l+1) !}{(2 l-2 k) !} \sqrt{2 \pi} \frac{(2 l-2 k) !}{2^{l-k}(l-k) !}\left(\boldsymbol{e}^{-}-\boldsymbol{e}^{+}\right) \\
& =\sum_{l=k}^{\infty}(-1)^{l+k+1} \frac{\sqrt{2 \pi}}{(l+1) ! l !} \frac{(2 l+1) !}{2^{l-k}(l-k) !}\left(\boldsymbol{e}^{-}-\boldsymbol{e}^{+}\right) \\
& =\sqrt{2 \pi}\left(\begin{array}{c}
2 k+1 \\
k
\end{array}\right){ }_{1} F_{1}\left(k+\frac{3}{2} ; k+2 ;-2\right)\left(\boldsymbol{e}^{+}-\boldsymbol{e}^{-}\right) .
\end{aligned}
$$

After normalising, i.e. dividing by $\eta_{2 k}$, respectively $\eta_{2 k+1}$ and applying the conjugation $\dagger$, we can write 
Proposition 4.1. The discrete function $\delta_{0}$ can be written as a linear combination of discrete Hermite functions, i.e. $\delta_{0}=\sum_{n \in \mathbb{Z}} H_{n}(\xi) d_{n}^{0}[1]$, with coefficients $d_{n}^{0}$

$$
\begin{aligned}
d_{2 k}^{0} & =\frac{1}{(k !)^{2}}{ }_{1} F_{1}\left(k+\frac{1}{2} ; k+1 ;-2\right), \\
d_{2 k+1}^{0} & =\frac{1}{k !(k+1) !}{ }_{1} F_{1}\left(k+\frac{3}{2} ; k+2 ;-2\right)\left(\boldsymbol{e}^{-}-\boldsymbol{e}^{+}\right) .
\end{aligned}
$$

This generalized hypergeometric series is also known as (Kummer's) confluent hypergeometric function of the first kind (See e.g. [18]), which converges everywhere. In general, the hypergeometric function ${ }_{p} F_{q}(a ; b ; z)$ with $p \leq q$ converges for all finite values of $z$ and defines an entire function, [19, Sec. 16.2]. We have that

$$
\lim _{k \rightarrow \infty}{ }_{1} F_{1}\left(k+\frac{1}{2} ; k+1 ;-2\right)=\lim _{k \rightarrow \infty}{ }_{1} F_{1}\left(k+\frac{3}{2} ; k+2 ;-2\right)=e^{-2},
$$

so that

$$
\lim _{k \rightarrow \infty} d_{2 k}^{0}=\lim _{k \rightarrow \infty} d_{2 k+1}^{0}=0 .
$$

The same question can be asked for any other $\delta_{j}, j \in \mathbb{Z}$ and the reasoning will be completely analoguous. The Taylor series are given by

$$
\begin{aligned}
& \delta_{j}(x)=\sum_{l=j}^{\infty} \frac{(-1)^{l-j}}{(l-j) !(l+j) !} \xi^{2 l}[1](x)+ \\
& \sum_{l=j-1}^{\infty} \frac{(-1)^{l-j+1}}{(l-j+1) !(l+j) !} \xi^{2 l+1}[1](x) \boldsymbol{e}^{+}+\sum_{l=j}^{\infty} \frac{(-1)^{l-j}}{(l-j) !(l+j+1) !} \xi^{2 l+1}[1](x) \boldsymbol{e}^{-}
\end{aligned}
$$

for positive $j$ and

$$
\begin{aligned}
& \delta_{j}(x)=\sum_{l=|j|}^{\infty} \frac{(-1)^{l-j}}{(l-j) !(l+j) !} \xi^{2 l}[1](x)+ \\
& \sum_{l=|j|}^{\infty} \frac{(-1)^{l-j+1}}{(l-j+1) !(l+j) !} \xi^{2 l+1}[1](x) \boldsymbol{e}^{+}+\sum_{l=|j|-1}^{\infty} \frac{(-1)^{l-j}}{(l-j) !(l+j+1) !} \xi^{2 l+1}[1](x) \boldsymbol{e}^{-}
\end{aligned}
$$

for negative $j$. 
Let us calculate the inner product for $j>0$ :

$$
\begin{aligned}
\left\langle H_{2 k}(\xi) G,[1] \delta_{j}^{\dagger}\right\rangle= & \sum_{l=j}^{\infty} \frac{(-1)^{l-j}}{(l-j) !(l+j) !}\left\langle H_{2 k}(\xi) G,[1]\left(\xi^{2 l}\right)^{\dagger}\right\rangle \\
= & \sum_{l=j}^{\infty} \frac{(-1)^{l-j}}{(l-j) !(l+j) !}\left\langle(-1)^{k} \partial^{2 k} G, \xi^{2 l}[1]\right\rangle \\
= & \sum_{l=j}^{\infty} \frac{(-1)^{l+k-j}}{(l-j) !(l+j) !}\left\langle G, \xi^{2 l}[1]\left(\partial^{2 k}\right)^{\dagger}\right\rangle \\
= & \sum_{l=\max (k, j)}^{\infty} \frac{(-1)^{l+k-j}}{(l-j) !(l+j) !} \frac{(2 l) !}{(2 l-2 k) !}\left\langle G, \xi^{2 l-2 k}[1]\right\rangle \\
= & \sum_{l=\max (k, j)}^{\infty} \frac{(-1)^{l+k-j}}{(l-j) !(l+j) !} \frac{(2 l) !}{(2 l-2 k) !} \sqrt{2 \pi} \frac{(2 l-2 k) !}{2^{l-k}(l-k) !} \\
= & \frac{(-1)^{\mu-j+k} \sqrt{2 \pi} 2^{k-\mu}(2 \mu) !}{(\mu-j) !(\mu+j) !(\mu-k) !} \\
& \times{ }_{3} F_{3}\left(1, \mu+\frac{1}{2}, \mu+1 ; \mu-j+1, \mu+j+1, \mu-k+1 ;-2\right) .
\end{aligned}
$$

In the last line, we have set $\mu:=\max (k, j)$. Note that this generalized hypergeometric function will simplify to a ${ }_{2} F_{2}$ in both cases $\mu=k$ and $\mu=j$.

For the odd Hermite polynomials, we have that (let $\mu:=\max (k, j)$ and $\mu^{\prime}:=$ $\max (k, j-1))$ 


$$
\begin{aligned}
& \left\langle H_{2 k+1}(\xi) G,[1] \delta_{j}^{\dagger}\right\rangle \\
& =\left\langle(-1)^{k+1} \partial^{2 k+1} G,[1] \sum_{l=j-1}^{\infty} \frac{(-1)^{l-j+1}}{(l-j+1) !(l+j) !}\left(\xi^{2 l+1}\right)^{\dagger}\right\rangle \boldsymbol{e}^{-} \\
& +\left\langle(-1)^{k+1} \partial^{2 k+1} G,[1] \sum_{l=j}^{\infty} \frac{(-1)^{l-j}}{(l-j) !(l+j+1) !}\left(\xi^{2 l+1}\right)^{\dagger}\right\rangle \boldsymbol{e}^{+} \\
& =\sum_{l=j-1}^{\infty} \frac{(-1)^{l-j+k+1}}{((l-j+1) !(l+j) !}\left\langle G, \partial^{2 k+1} \xi^{2 l+1}[1]\right\rangle e^{-} \\
& +\sum_{l=j}^{\infty} \frac{(-1)^{l-j+k}}{((l-j) !(l+j+1) !}\left\langle G, \partial^{2 k+1} \xi^{2 l+1}[1]\right\rangle \boldsymbol{e}^{+} \\
& =\sum_{l=\mu^{\prime}}^{\infty} \frac{(-1)^{l-j+k+1}(2 l+1) !}{(l-j+1) !(l+j) !(2 l-2 k) !}\left\langle G, \xi^{2 l-2 k}[1]\right\rangle e^{-} \\
& +\sum_{l=\mu}^{\infty} \frac{(-1)^{l-j+k}(2 l+1) !}{(l-j) !(l+j+1) !(2 l-2 k) !}\left\langle G, \xi^{2 l-2 k}[1]\right\rangle \boldsymbol{e}^{+} \\
& =\sum_{l=\mu^{\prime}}^{\infty} \frac{(-1)^{l-j+k+1}(2 l+1) !}{(l-j+1) !(l+j) !(2 l-2 k) !} \frac{\sqrt{2 \pi}(2 l-2 k) !}{2^{l-k}(l-k) !} e^{-}+ \\
& +\sum_{l=\mu}^{\infty} \frac{(-1)^{l-j+k}(2 l+1) !}{(l-j) !(l+j+1) !(2 l-2 k) !} \frac{\sqrt{2 \pi}(2 l-2 k) !}{2^{l-k}(l-k) !} e^{+} \\
& =\frac{\sqrt{2 \pi}(-1)^{1-j+k+\mu^{\prime}} 2^{k-\mu^{\prime}}\left(1+2 \mu^{\prime}\right) !}{\left(1-j+\mu^{\prime}\right) !\left(j+\mu^{\prime}\right) !\left(\mu^{\prime}-k\right) !} \\
& \times{ }_{3} F_{3}\left(1,1+\mu^{\prime}, \frac{3}{2}+\mu^{\prime} ; 2-j+\mu^{\prime}, 1+j+\mu^{\prime}, 1-k+\mu^{\prime} ;-2\right) \boldsymbol{e}^{-} \\
& +\frac{\sqrt{2 \pi}(-1)^{-j+k+\mu} 2^{k-\mu}(1+2 \mu) !}{(1+j+\mu) !(\mu-j) !(\mu-k) !} \\
& \times_{3} F_{3}\left(1,1+\mu, \frac{3}{2}+\mu ; 1-j+\mu, 2+j+\mu, 1-k+\mu ;-2\right) \boldsymbol{e}^{+} .
\end{aligned}
$$

The results for $j<0$ are completely analoguous, and it is immediately clear from the Taylor series that coefficients of $\boldsymbol{e}^{+}$and $\boldsymbol{e}^{-}$will be interchanged. Also here, this generalized hypergeometric will simplify to a ${ }_{2} F_{2}$ in both cases $\mu=k$ and $\mu=j$. For both inner products above, we end by normalizing $H_{2 k}$ and $H_{2 k+1}$ to obtain:

Proposition 4.2. The discrete function $\delta_{j}, j>0$, can be written as a linear combination of discrete Hermite functions, i.e. $\delta_{j}=\sum_{k \in \mathbb{Z}} H_{k}(\xi) d_{k}^{j}[1]$, with 
coefficients $d_{k}^{j}$ given by

$$
\begin{aligned}
& d_{2 k}^{j}=\frac{(-1)^{\mu-j+k} 2^{k-\mu}(2 \mu) !}{(2 k) !(\mu-j) !(\mu+j) !(\mu-k) !} \\
& \times{ }_{3} F_{3}\left(1, \mu+\frac{1}{2}, \mu+1 ; \mu-j+1, \mu+j+1, \mu-k+1 ;-2\right), \\
& d_{2 k+1}^{j}=\frac{(-1)^{1-j+k+\mu^{\prime}} 2^{k-\mu^{\prime}}\left(1+2 \mu^{\prime}\right) !}{\left(1-j+\mu^{\prime}\right) !\left(j+\mu^{\prime}\right) !\left(\mu^{\prime}-k\right) !(2 k+1) !} \\
& \times{ }_{3} F_{3}\left(1,1+\mu^{\prime}, \frac{3}{2}+\mu^{\prime} ; 2-j+\mu^{\prime}, 1+j+\mu^{\prime}, 1-k+\mu^{\prime} ;-2\right) \boldsymbol{e}^{+} \\
& +\frac{(-1)^{-j+k+\mu} 2^{k-\mu}(1+2 \mu) !}{(1+j+\mu) !(\mu-j) !(\mu-k) !(2 k+1) !} \\
& \times{ }_{3} F_{3}\left(1,1+\mu, \frac{3}{2}+\mu ; 1-j+\mu, 2+j+\mu, 1-k+\mu ;-2\right) e^{-} .
\end{aligned}
$$

If $j<0$,

$$
\begin{aligned}
d_{2 k}^{j}= & \frac{(-1)^{\mu-j+k} 2^{k-\mu}(2 \mu) !}{(2 k) !(\mu-j) !(\mu+j) !(\mu-k) !} \\
& \quad \times{ }_{3} F_{3}\left(1, \mu+\frac{1}{2}, \mu+1 ; \mu-j+1, \mu+j+1, \mu-k+1 ;-2\right), \\
d_{2 k+1}^{j}= & \frac{(-1)^{1-j+k+\mu} 2^{k-\mu}(1+2 \mu) !}{(1-j+\mu) !(j+\mu) !(\mu-k) !(2 k+1) !} \\
& \quad \times{ }_{3} F_{3}\left(1,1+\mu, \frac{3}{2}+\mu ; 2-j+\mu, 1+j+\mu, 1-k+\mu ;-2\right) \boldsymbol{e}^{+} \\
+ & \frac{(-1)^{-j+k+\mu} 2^{k-\mu}(1+2 \mu) !}{(1+j+\mu) !(\mu-j) !(\mu-k) !(2 k+1) !} \\
& \quad \times{ }_{3} F_{3}\left(1,1+\mu, \frac{3}{2}+\mu ; 1-j+\mu, 2+j+\mu, 1-k+\mu ;-2\right) \boldsymbol{e}^{-} .
\end{aligned}
$$

We used the notation $\mu=\max (k,|j|)$ and $\mu^{\prime}=\max (k,|j|-1)$.

For the special case $j=0$, we reobtain the result from proposition 4.1. To avoid confusion, we will always write $j$ in uppercase of the coefficients $d_{n}^{j}$, also for $\delta_{0}$.

For the limit $\lim _{n \rightarrow \infty} d_{n}^{j}$, we need to investigate the corresponding limit of the generalized hypergeometric function. As $k \rightarrow \infty, \mu=k$ and we need

$$
\lim _{k \rightarrow \infty}{ }_{2} F_{2}\left(k+\frac{1}{2}, k+1 ; k-j+1, k+j+1 ;-2\right)
$$

and

$$
\lim _{k \rightarrow \infty}{ }_{2} F_{2}\left(k+\frac{3}{2}, k+1 ; k-j+1, k+j+1 ;-2\right) .
$$


The asymptotic behaviour of this expression is the series ${ }_{0} F_{0}(; ; z)=e^{z}$, so that these limits equal $e^{-2}$. It follows immediately that

$$
\lim _{n \rightarrow \infty} d_{n}^{j}=0 .
$$

\subsection{Evaluation method}

From [11], derivatives of $\delta_{j}$ can be found in .

$$
\begin{aligned}
\partial^{2 k} \delta_{j} & =\sum_{i=0}^{2 k}(-1)^{i}\left(\begin{array}{c}
2 k \\
i
\end{array}\right) \delta_{j-(k-i)}, \\
\partial^{2 k+1} \delta_{j} & =\sum_{i=0}^{2 k+1}(-1)^{i}\left(\begin{array}{c}
2 k+1 \\
i
\end{array}\right)\left(\delta_{j-(k+1-i)} \boldsymbol{e}^{+}+\delta_{j-(k-i)} \boldsymbol{e}^{-}\right) .
\end{aligned}
$$

We will now use these to evaluate the action of the Hermite operators on the Gauss distribution $G$. For the action of $H_{2 k}$; we get

$$
\begin{aligned}
H_{2 k} G & =(-1)^{k} \partial^{2 k} \sqrt{2 \pi} e^{\frac{\partial^{2}}{2}} \delta_{0}=\sum_{l=0}^{\infty}(-1)^{k} \partial^{2 k} \sqrt{2 \pi} \frac{\partial^{2 l}}{2^{\ell} l !} \delta_{0} \\
& =\sum_{l=0}^{\infty} \sqrt{2 \pi} \frac{(-1)^{k}}{2^{\ell} l !} \partial^{2(l+k)} \delta_{0} \\
& =\sum_{l=0}^{\infty} \sqrt{2 \pi} \frac{(-1)^{k}}{2^{\ell} l !}\left(\sum_{i=0}^{2(l+k)}(-1)^{i}\left(\begin{array}{c}
2 l+2 k \\
i
\end{array}\right) \delta_{-(l+k)+i}\right) .
\end{aligned}
$$

To obtain the coefficients in $\delta_{0}$, we look at the coefficients $-(l+k)+i=0$, or $i=k+l$ :

$$
\sum_{l=0}^{\infty} \sqrt{2 \pi} \frac{(-1)^{\ell}}{2^{\ell} l !}\left(\begin{array}{c}
2 l+2 k \\
k+l
\end{array}\right)=\sqrt{2 \pi}\left(\begin{array}{c}
2 k \\
k
\end{array}\right){ }_{1} F_{1}\left(k+\frac{1}{2} ; k+1 ;-2\right) .
$$


For the case of odd Hermite polynomials, we get

$$
\begin{aligned}
H_{2 k+1} G= & (-1)^{k+1} \partial^{2 k+1} \sqrt{2 \pi} e^{\left(\partial^{2} / 2\right)} \delta_{0}=\sum_{l=0}^{\infty}(-1)^{k+1} \partial^{2 k+1} \sqrt{2 \pi} \frac{\partial^{2 l}}{2^{\ell} l !} \\
= & \sum_{l=0}^{\infty} \sqrt{2 \pi} \frac{(-1)^{k+1}}{2^{\ell} l !} \partial^{2(l+k)+1} \delta_{0} \\
= & \sum_{l=0}^{\infty} \sqrt{2 \pi} \frac{(-1)^{k+1}}{2^{\ell} l !}\left(\sum_{i=0}^{2(l+k)+1}(-1)^{i}\left(\begin{array}{c}
2 l+2 k+1 \\
i
\end{array}\right) \delta_{-(l+k+1-i)} e^{+}\right) \\
& +\sum_{l=0}^{\infty} \sqrt{2 \pi} \frac{(-1)^{k+1}}{2^{\ell} l !}\left(\sum_{i=0}^{2(l+k)+1}(-1)^{i}\left(\begin{array}{c}
2 l+2 k+1 \\
i
\end{array}\right) \delta_{-(l+k-i)} e^{-}\right)
\end{aligned}
$$

We look for the coefficients in $\delta_{0}$ : for $\boldsymbol{e}^{+}, i=k+1+l$, while for $\boldsymbol{e}^{-}, i=k+l$.

$$
\begin{aligned}
H_{2 k+1} G(0)= & \sum_{l=0}^{\infty} \sqrt{2 \pi} \frac{(-1)^{k+1}}{2^{\ell} l !} \times \\
& \left((-1)^{k+l+1}\left(\begin{array}{c}
2 l+2 k+1 \\
k+l+1
\end{array}\right) \boldsymbol{e}^{+}+(-1)^{l+k}\left(\begin{array}{c}
2 l+2 k+1 \\
l+k
\end{array}\right) \boldsymbol{e}^{-}\right) \\
= & \sum_{l=0}^{\infty} \sqrt{2 \pi} \frac{(-1)^{l}}{2^{\ell} l !}\left(\left(\begin{array}{c}
2 l+2 k+1 \\
k+l+1
\end{array}\right) \boldsymbol{e}^{+}-\left(\begin{array}{c}
2 l+2 k+1 \\
l+k
\end{array}\right) \boldsymbol{e}^{-}\right) \\
= & \sum_{l=0}^{\infty} \sqrt{2 \pi} \frac{(-1)^{l}}{2^{\ell} l !}\left(\begin{array}{c}
2 l+2 k+1 \\
k+l
\end{array}\right)\left(\boldsymbol{e}^{+}-\boldsymbol{e}^{-}\right) \\
= & \sqrt{2 \pi}\left(\begin{array}{c}
2 k+1 \\
k
\end{array}\right){ }_{1} F_{1}\left(k+\frac{3}{2} ; k+2 ;-2\right)\left(\boldsymbol{e}^{+}-\boldsymbol{e}^{-}\right) .
\end{aligned}
$$

These are indeed the same result as in 4.1.

For $\delta_{j}$, we can do similar calculations. For the even Hermite functions, we are now interested in $\delta_{j}$, hence in coefficients for $i=j+l+k$ :

$$
\sum_{l=|j|-k}^{\infty} \sqrt{2 \pi} \frac{(-1)^{l+j}}{2^{l} l !}\left(\begin{array}{c}
2 l+2 k \\
j+k+l
\end{array}\right) \delta_{j} .
$$

Since $0 \leq i \leq 2 l+2 k$, also $0 \leq j+k+l \leq 2 l+2 k$, or $|j| \leq l+k$. This value is

$$
\sqrt{2 \pi}(-1)^{k} 2^{k-j} F_{2}\left(j+1, j+\frac{1}{2} ; 1+2 j, j-k+1 ;-2\right)
$$

in $x=j$.

For the odd case, we set $i=j+l+k+1$ for $\boldsymbol{e}^{+}$and $i=j+k+l$ for $\boldsymbol{e}^{-}$. This implies that $-k-l-1 \leq j \leq l+k$ and $-k-l \leq j \leq k+l+1$. The coefficient 
in $\delta_{j}$ is

$$
\begin{gathered}
\sum_{l=\max (j-k, 0)}^{\infty} \sqrt{2 \pi} \frac{(-1)^{k+1}}{2^{\ell} l !}(-1)^{j+l+k+1}\left(\begin{array}{c}
2 k+2 l+1 \\
j+k+l+1
\end{array}\right) e^{+} \\
-\sum_{l=\max (j-k-1,0)}^{\infty} \sqrt{2 \pi} \frac{(-1)^{k+1}}{2^{\ell} l !}(-1)^{j+l+k}\left(\begin{array}{c}
2 l+2 k+1 \\
j+k+l
\end{array}\right) e^{-} \\
=\sum_{l=\max (j-k, 0)}^{\infty} \sqrt{2 \pi} \frac{(-1)^{l+j}}{2^{\ell} l !}\left(\begin{array}{c}
2 k+2 l+1 \\
j+k+l+1
\end{array}\right) e^{+} \\
\quad-\sum_{l=\max (j-k-1,0)}^{\infty} \sqrt{2 \pi} \frac{(-1)^{l+j+1}}{2^{\ell} l !}\left(\begin{array}{c}
2 l+2 k+1 \\
j+k+l
\end{array}\right) \boldsymbol{e}^{-} .
\end{gathered}
$$

This result equals $\left(\mu=\max (j-k, 0), \mu^{\prime}=\max (j-k-1,0)\right)$

$$
\begin{aligned}
& \sqrt{2 \pi} \frac{(-1)^{j+\mu}}{2^{\mu} \mu !}\left(\begin{array}{c}
2 k+2 \mu+1 \\
j+k+\mu+1
\end{array}\right) \\
& \quad \times{ }_{3} F_{3}\left(1, k+\mu+1, k+\mu+\frac{3}{2} ; \mu+1,-j+k+\mu+1, j+k+\mu+2 ;-2\right) e^{+} \\
& +\sqrt{2 \pi} \frac{(-1)^{j+\mu^{\prime}+1}}{2^{\mu^{\prime}} \mu^{\prime} !}\left(\begin{array}{c}
2 k+2 \mu^{\prime}+1 \\
j+k+\mu^{\prime}
\end{array}\right) \\
& \quad \times{ }_{3} F_{3}\left(1, k+\mu^{\prime}+1, k+\mu^{\prime}+\frac{3}{2} ; \mu^{\prime}+1,-j+k+\mu^{\prime}+2, j+k+\mu^{\prime}+1 ;-2\right) e^{-} .
\end{aligned}
$$

We now have another expression for the coefficients $d_{n}^{j}$, after normalizing by $\eta_{2 k}$, respectively $\eta_{2 k+1}$ :

Theorem 4.3. The discrete function $\delta_{j}, j>0$, can be written as a linear combination of discrete Hermite functions, i.e. $\delta_{j}=\sum_{k \in \mathbb{Z}} H_{k}(\xi) d_{k}^{j}[1]$, with coefficients $d_{k}^{j}$

$$
\begin{aligned}
d_{2 k}^{j}= & \frac{(-1)^{k} 2^{k-j}}{(2 k) !}{ }_{2} F_{2}\left(j+1, j+\frac{1}{2} ; 1+2 j, j-k+1 ;-2\right) \\
d_{2 k+1}^{j}= & \frac{(-1)^{j+\mu}}{2^{\mu} \mu !(2 k+1) !}\left(\begin{array}{c}
2 k+2 \mu+1 \\
j+k+\mu+1
\end{array}\right) \\
& { }_{3} F_{3}\left(1, k+\mu+1, k+\mu+\frac{3}{2} ; \mu+1,-j+k+\mu+1, j+k+\mu+2 ;-2\right) e^{+} \\
+ & \frac{(-1)^{j+\mu^{\prime}+1}}{2^{\mu^{\prime}} \mu^{\prime} !(2 k+1) !}\left(\begin{array}{c}
2 k+2 \mu^{\prime}+1 \\
j+k+\mu^{\prime}
\end{array}\right) \\
& { }_{3} F_{3}\left(1, k+\mu^{\prime}+1, k+\mu^{\prime}+\frac{3}{2} ; \mu^{\prime}+1,-j+k+\mu^{\prime}+2, j+k+\mu^{\prime}+1 ;-2\right) e^{-}
\end{aligned}
$$

It is easily checked that the expressions in propositions 4.2 and 4.3 are equal for concrete values of $j$ and $k$. 


\subsection{Examples and conclusion}

To give some examples, the coefficients $d_{n}^{0}, d_{n}^{3}, d_{n}^{-3}, d_{n}^{8}, d_{n}^{30}$ and $d_{n}^{35}$ are plotted in Figure 1. On the $x$-axis, the $n$-th Hermite polynomial is represented, while on the $y$-axis, the respective coefficients are depicted. For $n$ even, the values of the delta-coefficients are always scalar and plotted in red. For $n$ odd, the values are vectorial: in green, one finds the coefficents for $\boldsymbol{e}^{+}$, in blue, we find the coefficients for $\boldsymbol{e}^{-}$.

In particular, for $\delta_{0}$, the odd coefficients are equal for $\boldsymbol{e}^{+}$and $\boldsymbol{e}^{-}$up to a minus sign, i.e. $d_{2 k+1}^{0}=r\left(\boldsymbol{e}^{-}-\boldsymbol{e}^{+}\right)$, with $r$ scalar. This $r$ is also depicted in red.

In the plots of $d_{n}^{3}$ and $d_{n}^{-3}$, it is clear that the coefficients of $\boldsymbol{e}^{ \pm}$reflected with respect to the $x$-axis gives the coefficients of $e^{\mp}$ of the delta function with negated index.

The absolute values of all coefficients $d_{n}^{j}$ converge to 0 for fixed $j$ if $n$ enlarges, as was already mentioned in (4.3).

Having found the coefficients of the $\delta_{j}$ functions with respect to the discrete Hermite polynomials in the discrete Weierstrass space, we can now calculate the Weierstrass transform of these delta functions. Consider the most simple case $\delta_{0}=\sum_{n \in \mathbb{N}} H_{n}(\xi) d_{n}^{0}$ :

$$
\begin{aligned}
\mathcal{W}\left[\delta_{0}(\xi)[1]\right](z)= & \sum_{n \in \mathbb{N}} \mathcal{W}\left[H_{n}(\xi)\right](z) d_{n}^{0}=\sum_{n \in \mathbb{N}} d_{n}^{0}(-1)^{\left\lfloor\frac{n}{2}\right\rfloor} z^{n} \\
= & \sum_{k=0}^{\infty}(-1)^{k}\left(\frac{1}{(k !)^{2}}{ }_{1} F_{1}\left(k+\frac{1}{2} ; k+1 ;-2\right) z^{2 k}\right. \\
& \left.\quad+\frac{1}{k !(k+1) !}{ }_{1} F_{1}\left(k+\frac{3}{2} ; k+2 ;-2\right)\left(\boldsymbol{e}^{-}-\boldsymbol{e}^{+}\right) z^{2 k+1}\right) .
\end{aligned}
$$

For every $z \in \mathbb{C}$, this number is finite. However, $\lim _{|z| \rightarrow \infty} \mathcal{W}\left[\delta_{0}(\xi)[1]\right](z)=$ $+\infty$, indicating that this function is unbounded and will certainly not converge to the Gaussian kernel, as is the case in the continuous case. As $\delta_{0}$ is not a continuous function, it may not be surprising that its Weierstrass transform does not have nice behaviours at infinity. In analogy, it was already found out in [20] that also the CK-extension of $\delta_{0}$ 'explodes' at infinity. An expected result would be that the Weierstrass transform of the discrete delta distribution $\boldsymbol{\delta}_{0}$ is the Gaussian function. However, this is only a presumption, which needs to be carefully investigated.

\section{Discrete Weierstrass space, revised}

Let us now come back to the question how we can describe the elements in the discrete Weierstrass space $\mathcal{W}$. Take an element $f \in \mathcal{W}$ and consider $f G$. On 
Figure 1: Plots of $\delta$-coefficients

(a) Coefficients $d_{n}^{0}$

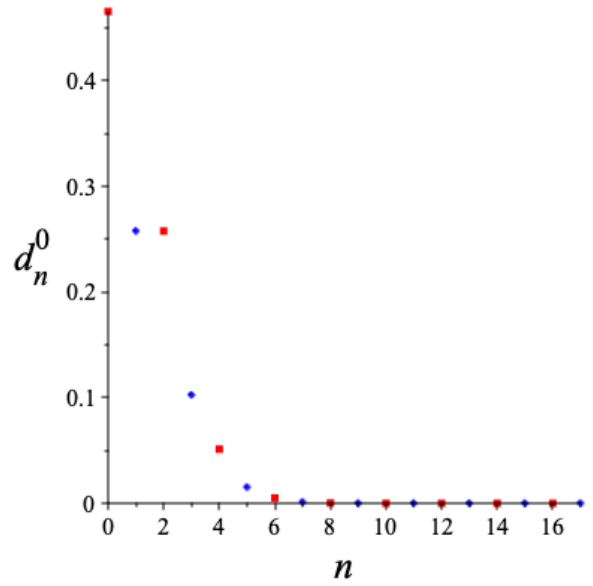

(c) Coefficients $d_{n}^{-3}$

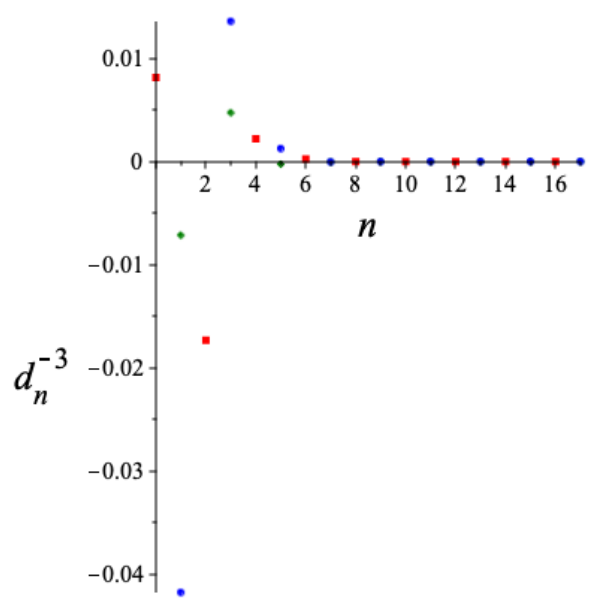

(b) Coefficients $d_{n}^{3}$

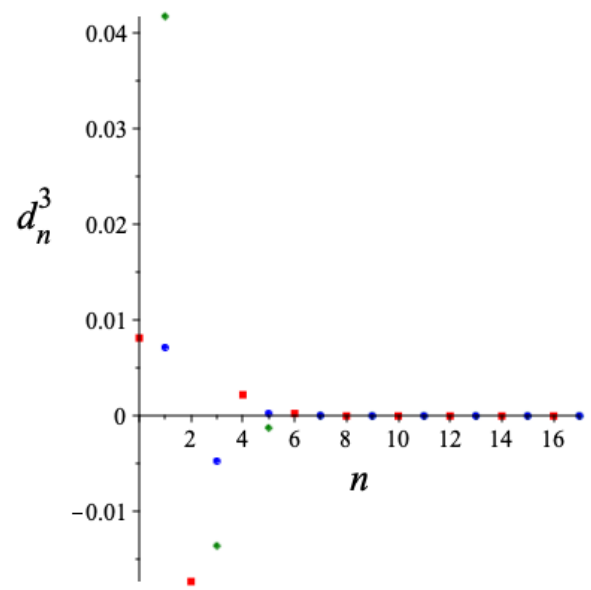

(d) Coefficients $d_{n}^{8}$

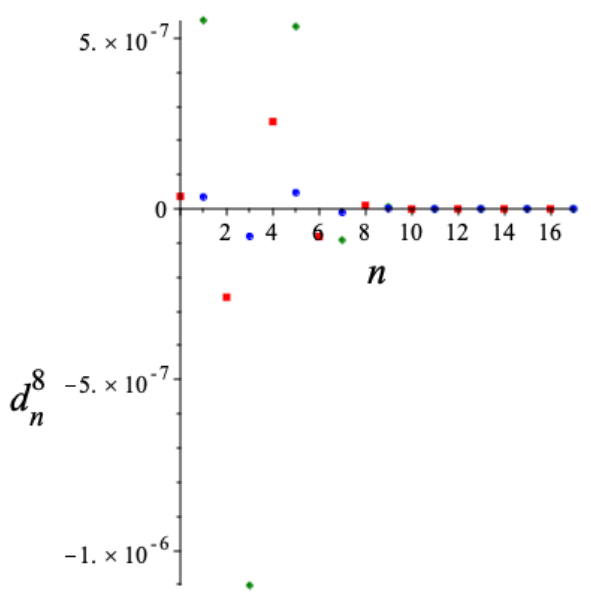


(a) Coefficients $d_{n}^{30}$

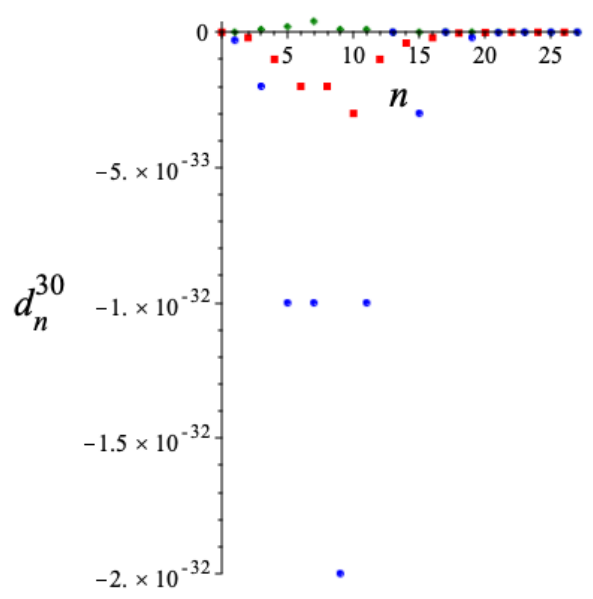

(b) Coefficients $d_{n}^{35}$

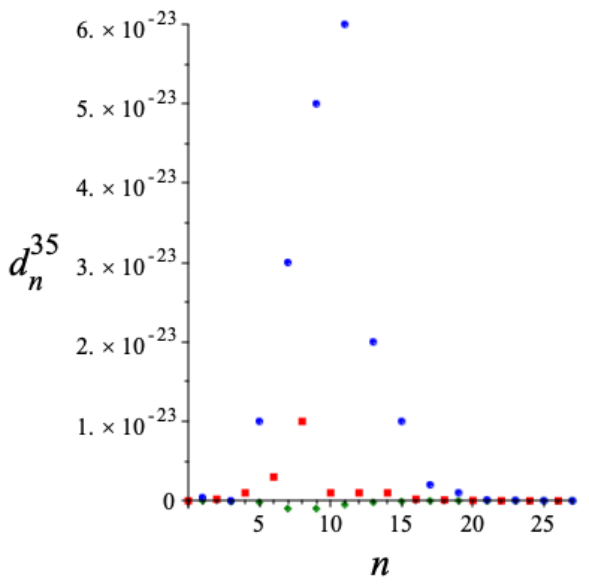

the one hand, $f G$ acts on functions in $\mathcal{W}$ in particular $f G$ acts on polynomials, by means of the inner product (3.2). It means $f G$ is a distribution. On the other hand, as the delta functions $\delta_{j} \in \mathcal{W}, f G$ acts on $\delta_{j}$, which is pointwise evaluation of $f G$. This means $f G$ is a function on $\mathbb{Z}$. With every distribution $f G$, there is associated a density function on $\mathbb{Z}$, which reflects the dual aspect in this theory. In this way, $\mathcal{W} G$ can be seen to contain both functions and distributions and thus is a subspace of $\mathcal{D} \cap \mathcal{F}$, where $\mathcal{D}$ and $\mathcal{F}$ are the spaces of distributions and functions, respectively.

An element $f \in \mathcal{W}$ is not a distribution: it does not act on polynomials (it has no compact support). In particular, polynomials, which are in $\mathcal{W}$, are no distributions. Nor every element of $\mathcal{W}$ is a function:

Example 1. Take $f \in \mathcal{W}$, say $f=\sum_{n \in \mathbb{N}} \frac{H_{n}}{\sqrt{\eta_{n}}} c_{n},\left(c_{n}\right)_{N} \subset \mathbb{R}$ and suppose $f$ is a function. Then it must have a Taylor series expansion. Evaluation of $f$ in zero gives us the constant term in this series. In particular, $f(0)=\left.f(\xi)\right|_{\xi=0}$ is of the form $\sum_{n \in \mathbb{N}} \frac{H_{n}(0)}{\sqrt{\eta_{n}}} c_{n} . \quad f$ being an element of $\mathcal{W}$, it must hold that $\langle f, f\rangle=\sum_{n \in \mathbb{N}} c_{n}^{2}<\infty$, hence the coefficients $\left(c_{n}\right)_{n} \in \ell_{2}(\mathbb{R})$. Let us first consider the behaviour of $\sum_{k \in \mathbb{N}} \frac{H_{2 k}(0)}{\sqrt{\eta_{2 k}}}$ (remark that $H_{2 l+1}(0)=0, \forall l \in \mathbb{N}$, as 
constant coefficients only occur in even Hermite polynomials):

$$
\begin{aligned}
\frac{H_{2 k}(0)}{\sqrt{\eta_{2 k}}} & =\frac{(2 k) !}{k ! 2^{k} \sqrt{\sqrt{2 \pi}}} \\
& =\frac{\sqrt{(2 k) !}}{2^{k} k !(2 \pi)^{1 / 4}} \\
& \geq \frac{\sqrt{\sqrt{2 \pi}(2 k)^{2 k+\frac{1}{2}} e^{-2 k}}}{(2 \pi)^{1 / 4} 2^{k} k^{k+1 / 2} e^{-k+1}} \\
& =\frac{(2 k)^{k+\frac{1}{4} e^{-k}}}{2^{k} e^{-k+1}}=\frac{2^{\frac{1}{4}}}{e} k^{-\frac{1}{4}} \approx k^{-\frac{1}{4}},
\end{aligned}
$$

where we made use of Stirlings asymptotic behaviour. By multiplying this series with $\left(c_{k}\right)_{k}=\left(\frac{1}{(2 k)^{s}}\right)_{k}, s>\frac{1}{2}$, one gets $\sum_{k=0}^{\infty} \frac{1}{k^{s+\frac{1}{4}}}$. This diverges if $s<\frac{3}{4}$.

We can conclude that not every converging combination of Hermite polynomials with coefficients in $\ell_{2}$ is a function, meaning pointwise convergence is not always implied. This counterexample proofs that $\mathcal{W}$ is not a subspace of $\mathcal{F}$.

\section{Conclusion}

We established a discrete version of the Weierstrass transform in a discrete Hermitian Clifford setting, based on the discrete Gauss distribution $G$. Associated with that, we constructed a space of discrete 'functions' for which this Weierstrass transform makes sense. The discrete Weierstrass space fullfills our needs: its basis is given by the discrete Hermite polynomials and a sesquilinear form is given by $(P, R)=\left[\left\langle P(\xi) G,[1](R(\xi))^{\dagger}\right\rangle\right]_{0}$, where $P$ and $Q$ are elements of the Weierstrass space $\mathcal{W}$. We proved that the delta functions $\delta_{j}$ are elements of this space, by finding explicit formula for the coefficients $d_{k}^{j}$ in the expression $\sum_{n \in \mathbb{N}} H_{k} d_{k}^{j}$.

Extension of this construction to the discrete Weierstrass transform to higher dimensions $(m>1)$ as well as an analogue for discrete distributions, will be carried out in a next paper.

Furthermore, it is clear that a numerical analysis approach to the proposed construction may be carried out. This could also be the subject of another paper.

\section{Bibliography}

[1] G.G. Bilodeau, The Weierstrass transform and Hermite polynomials, Duke Math. J. 29 (2), pp. 293-308, 1962. 
[2] H. De Schepper, F. Sommen, Introductory clifford analysis, Operator theory, pp. 1339-1367, Springer Basel, 2015.

[3] N. Faustino, Discrete Clifford analysis (Doctoral dissertation), Universidade de Aveiro, Aveiro, 2009.

[4] H. De Schepper, F. Sommen, L. Van de Voorde. A basic framework for discrete Clifford analysis, Experiment. Math. 18 (4), pp. 385 - 395, 2009.

[5] K. Gürlebeck, A. Hommel, On finite difference potentials and their applications in a discrete function theory, Math. Meth. Appl. Sci. 25 (16-18), pp. 1563-1576, 2002.

[6] F. Baaske, S. Bernstein, H. De Ridder, F. Sommen, On solutions of a discretized heat equation in discrete Clifford analysis, J. Diff. Eq. Appl. 20 (2), pp. 271-295, 2013.

[7] K. Gürlebeck, W. Sprössig, Quaternionic analysis and elliptic boundary value problems, International Series of Numerical Mathematics 89 , Birkhauser Basel, 1989.

[8] N. Faustino, U. Kähler, F. Sommen, Discrete Dirac operators in Clifford analysis, Adv. Appl. Cliff. Alg. 17 (3), pp. 451-467, 2007.

[9] F. Brackx, H. De Schepper, F. Sommen, L. Van de Voorde Discrete Clifford analysis: an overview, CUBO - A Mathematical journal 11 (1), pp. 55-71, 2009.

[10] H. De Ridder, H. De Schepper, U. Kähler, F. Sommen. Discrete function theory based on skew Weyl relations, Proc. Amer. Math. Soc. 138 (9), pp. $3241-3256,2010$.

[11] H. De Bie, H. De Ridder, F. Sommen. Discrete Clifford Analysis: the one dimensional setting, Compl. Var. Ell. Eq. 57 (7-8), pp. 903-920, 2012.

[12] E. Norman, A Discrete Analogue of the Weierstrass Transform, Proceedings of the American Mathematical Society, 11(4), pp.596-604, 1960.

[13] S. Zhang, B. Harding, Discrete Weierstrass Fourier Transform and Experiments, arXiv:1502.07734, 2015.

[14] H. De Ridder, Discrete Clifford analysis: function theory and integral transforms (Doctoral dissertation), Ghent University, Faculty of Sciences, 2013.

[15] H. De Ridder, H. De Schepper, F. Sommen. Taylor series expansion in discrete Clifford analysis, Compl. Anal. Oper. Th. 8 (2), pp. 485 - 511, 2013.

[16] H. De Ridder, H. De Schepper, F. Sommen, Fueter polynomials in discrete Clifford analysis, Math. Zeit. 272 (1-2), pp. 253-268, 2012. 
[17] Wolfram Research, Inc., Mathematical Functions Site, http://functions.wolfram.com (05.01.23.0005.01, 05.01.23.0006.01, 05.01.23.0007.01), 2019 (accessed 25 May 2019).

[18] E.E. Kummer, De integralibus quibusdam definitis et seriebus infinitis, Journal fr die reine und angewandte Mathematik 17, pp228-242 (1837).

[19] F.W.J. Olver, A.B. Olde Daalhuis, D.W. Lozier, B.I. Schneider, R.F. Boisvert, C.W. Clark, B.R. Miller and B.V. Saunders, eds. NIST Digital Library of Mathematical Functions, http://dlmf.nist.gov/ (accessed 14 April 2019).

[20] H. De Ridder, H. De Schepper, F. Sommen, The Cauchy-Kovalevskaya extension theorem in discrete Clifford analysis, Communications on Pure \& Applied Analysis, 2011, 10 (4), pp. 1097-1109. 